\title{
Parabolic Equations on Uniformly Regular Riemannian Manifolds and Degenerate Initial Boundary Value Problems
}

\author{
Herbert Amann \\ Dedicated to Professor Yoshihiro Shibata on the occasion of his sixtieth birthday
}

\begin{abstract}
In this work there is established an optimal existence and regularity theory for second order linear parabolic differential equations on a large class of noncompact Riemannian manifolds. Then it is shown that it provides a general unifying approach to problems with strong degeneracies in the interior or at the boundary.
\end{abstract}

Mathematics Subject Classification (2010). Primary 58J35, 35K65; Secondary 53C20, 35K20.

Keywords. Noncompact Riemannian manifolds, linear parabolic boundary value problems, degenerate equations, weighted Sobolev spaces.

\section{Introduction}

This paper is devoted to second order initial boundary value problems for linear parabolic equations on a wide class of noncompact Riemannian manifolds, termed 'uniformly regular'. Important examples are complete Riemannian manifolds with no boundary and bounded geometry 11 In this setting there is already a rich theory for linear parabolic equations — predominantly heat equations - based on kernel estimates. Our main interest concerns, however, noncompact Riemannian manifolds with boundary for which very little is known so far (see the following sections for references). Prototypes of such cases are $m$-dimensional Riemannian submanifolds of $\mathbb{R}^{n}$ with compact boundary or funnel-like ends (cf. Examples 3.5).

In order to give the flavor of our main results we consider in this introduction a simplified version of the general problem. Namely, we restrict ourselves to autonomous equations with homogeneous boundary conditions.

\footnotetext{
${ }^{1}$ Precise definitions of and notations for all terms used in this introduction without further explanation are found in the following sections and the appendix.
} 
Let $M=(M, g)$ be a Riemannian manifold. We set

$$
\mathcal{A} u:=-\operatorname{div}(a \cdot \operatorname{grad} u),
$$

with $a$ being a symmetric positive definite $(1,1)$-tensor field on $M$ which is bounded and has bounded and continuous first order (covariant) derivatives. This is expressed by saying that $\mathcal{A}$ is a regular uniformly strongly elliptic differential operator.

We assume that $\partial_{0} M$ is open and closed in $\partial M$ and $\partial_{1} M:=\partial M \backslash \partial_{0} M$. Then we put

$$
\mathcal{B}_{0} u:=u \text { on } \partial_{0} M, \quad \mathcal{B}_{1} u:=(\nu \mid a \cdot \operatorname{grad} u) \text { on } \partial_{1} M,
$$

where these operators are understood in the sense of traces and $\nu$ is the inward pointing unit normal vector field on $\partial_{1} M$. Thus $\mathcal{B}:=\left(\mathcal{B}_{0}, \mathcal{B}_{1}\right)$ is the Dirichlet boundary operator on $\partial_{0} M$ and the Neumann operator on $\partial_{1} M$.

Throughout this paper, $0<T<\infty$ and $J:=[0, T]$. We write $M_{T}$ for the space time cylinder $M \times J$. Moreover, $\partial=\partial_{t}$ is the 'time derivative', $\partial M_{T}:=\partial M \times J$ the lateral boundary, and $M_{0}=M \times\{0\}$ the 'initial surface' of $M_{T}$. Then we consider the problem

$$
\partial u+\mathcal{A} u=f \text { on } M_{T}, \quad \mathcal{B} u=0 \text { on } \partial M_{T}, \quad u=u_{0} \text { on } M_{0} .
$$

The last equation is to be understood as $\gamma_{0} u=u_{0}$ with the 'initial' trace operator $\gamma_{0}$.

Of course, $\partial_{0} M$ or $\partial_{1} M$ or both may be empty. In such a situation obvious interpretations and modifications are to be applied.

We are interested in a strong $L_{p}$-theory for (1.2). To describe it we have to introduce (fractional order) Sobolev spaces. We always assume that $1<p<\infty$. The Sobolev space $W_{p}^{k}(M)$ is defined for $k \in \mathbb{N}$ to be the completion of $\mathcal{D}(M)$, the space of smooth functions with compact support, in $L_{1, \text { loc }}(M)$ with respect to the norm

$$
u \mapsto\left(\sum_{j=0}^{k}\left\|\left|\nabla^{j} u\right|_{g_{j}^{0}}\right\|_{L_{p}(M)}^{p}\right)^{1 / p} .
$$

Here $\nabla=\nabla_{g}$ is the Levi-Civita covariant derivative and $|\cdot|_{g_{j}^{0}}$ the $(0, j)$-tensor norm naturally induced by $g$. Thus $W_{p}^{0}(M)=L_{p}(M)$. Moreover,

$$
W_{p, \mathcal{B}}^{2}(M):=\left\{u \in W_{p}^{2}(M) ; \mathcal{B} u=0\right\} .
$$

We also need the space $W_{p}^{2-2 / p}(M)$ which is defined for $p \neq 2$ by real interpolation:

$$
W_{p}^{2-2 / p}(M):=\left(L_{p}(M), W_{p}^{2}(M)\right)_{1-1 / p, p} .
$$

Then

$$
W_{p, \mathcal{B}}^{2-2 / p}(M):=\left\{\begin{array}{lc}
\left\{u \in W_{p}^{2-2 / p}(M) ; \mathcal{B} u=0\right\}, & 3<p<\infty, \\
\left\{u \in W_{p}^{2-2 / p}(M) ; \mathcal{B}_{0} u=0\right\}, & 3 / 2<p<3, \\
W_{p}^{2-2 / p}(M), & 1<p<3 / 2 .
\end{array}\right.
$$


We set

$$
A:=\mathcal{A} \mid W_{p, \mathcal{B}}^{2}(M),
$$

considered as an unbounded linear operator in $L_{p}(M)$ with domain $W_{p, \mathcal{B}}^{2}(M)$. Then (1.2) can be expressed as an initial value problem for the evolution equation

$$
\dot{u}+A u=f \text { on } J, \quad u(0)=u_{0}
$$

in $L_{p}(M)$.

Now we are ready to formulate our main result in the present model setting. It is a special case of Theorem 3.4

Theorem 1.1. Let $M$ be a uniformly regular Riemannian manifold and let $p \notin\{3 / 2,3\}$. Suppose that $\mathcal{A}$ is regular and uniformly strongly elliptic. Then (1.2) has for each

$$
\left(f, u_{0}\right) \in L_{p}\left(J, L_{p}(M)\right) \times W_{p, \mathcal{B}}^{2-2 / p}(M)
$$

a unique solution

$$
u \in L_{p}\left(J, W_{p, \mathcal{B}}^{2}(M)\right) \cap W_{p}^{1}\left(J, L_{p}(M)\right) .
$$

The map $\left(f, u_{0}\right) \mapsto u$ is linear and continuous.

Equivalently: $-A$ generates an analytic semigroup on $L_{p}(M)$ and has the property of maximal regularity.

The finite time interval $J$ can be replaced by $\mathbb{R}^{+}$, provided we impose the additional assumption that the spectrum of $A$ is contained in $[\operatorname{Re} z \geq \gamma$ ] for some $\gamma>0$. This can always be achieved by replacing $A$ by $A+\omega$ for a sufficiently large $\omega>0$.

On the surface, this theorem looks exactly the same as the very classical existence and uniqueness theorem for second order parabolic equations on open subsets of $\mathbb{R}^{m}$ with smooth compact boundary (e.g., O.A. Ladyzhenskaya, V.A. Solonnikov, and N.N. Ural'ceva [27, Chapter IV] and R. Denk, M. Hieber, and J. Prüss [17]). However, it is in fact a rather deep-rooted vast generalization thereof since it applies to any uniformly regular Riemannian manifold.

Closely related to uniformly regular Riemannian manifolds are 'singular Riemannian manifolds' which are characterized by a 'singularity function' $\rho \in C^{\infty}(M,(0, \infty))$. More precisely, let $M=(M, g)$ be a Riemannian manifold and consider the conformal metric $\hat{g}:=g / \rho^{2}$ on $M$. Then the basic requirement for $M$ to be a singular Riemannian manifold is that $\hat{M}:=(M, \hat{g})$ be a uniformly regular Riemannian manifold. In Examples 4.1 we present some important instances of singular Riemannian manifolds, most notably the class of $m$-dimensional Riemannian submanifolds of $\mathbb{R}^{n}$ with finitely many cuspidal singularities.

By considering parabolic equations on singular Riemannian manifolds we are naturally led to study degenerate parabolic equations in weighted Sobolev spaces. To be more precise, we now assume that $M=(M, g)$ is a singular Riemannian manifold and $\rho \in C^{\infty}(M,(0, \infty))$ is a singularity function 
for it. Then $\mathcal{A}$ is said to be a $\rho$-regular uniformly $\rho$-elliptic differential operator if $\rho^{-2} a$ is symmetric, uniformly positive definite, and $\rho^{-2} a$ and $\rho^{-1} \nabla a$ are bounded and continuous. Note that this means that $\mathcal{A}$ is no longer uniformly strongly elliptic but that the ellipticity condition degenerates if $\rho$ tends to zero (or to infinity).

For $\lambda \in \mathbb{R}$ and $k \in \mathbb{N}$ we define the weighted Sobolev space $W_{p}^{k, \lambda}(M ; \rho)$ to be the completion of $\mathcal{D}(M)$ in $L_{1, \text { loc }}(M)$ with respect to the norm

$$
u \mapsto\left(\sum_{j=0}^{k}\left\|\rho^{\lambda+j}\left|\nabla^{j} u\right|_{g_{j}^{0}}\right\|_{L_{p}(M)}^{p}\right)^{1 / p} .
$$

Then

$$
W_{p}^{0, \lambda}(M ; \rho)=L_{p}^{\lambda}(M ; \rho):=\left\{u \in L_{p, \mathrm{loc}}(M) ; \rho^{\lambda} u \in L_{p}(M)\right\} .
$$

If $p \neq 2$, then

$$
W_{p}^{2-2 / p, \lambda}(M ; \rho):=\left(L_{p}^{\lambda}(M ; \rho), W_{p}^{2, \lambda}(M ; \rho)\right)_{1-1 / p, p} .
$$

Furthermore, $W_{p, \mathcal{B}}^{2, \lambda}(M ; \rho)$ and $W_{p, \mathcal{B}}^{2-2 / p, \lambda}(M ; \rho)$ are defined analogously to $W_{p, \mathcal{B}}^{2}(M)$ and $W_{p, \mathcal{B}}^{2-2 / p}(M)$, resp. Lastly, $W_{p}^{2}(M ; \rho):=W_{p}^{2,0}(M ; \rho)$, etc. Note that $L_{p}^{0}(M ; \rho)=L_{p}(M)$.

Using this we can now formulate our main result for degenerate parabolic equations in the present setting.

Theorem 1.2. Let $M$ be a singular Riemannian manifold, $\rho$ a singularity function for it, and $p \notin\{3 / 2,3\}$. Suppose $\mathcal{A}$ is $\rho$-regular and $\rho$-uniformly strongly elliptic. Then (1.2) has for each

$$
\left(f, u_{0}\right) \in L_{p}\left(J, L_{p}(M)\right) \times W_{p}^{2-2 / p, 2 / p}(M ; \rho)
$$

a unique solution

$$
u \in L_{p}\left(J, W_{p, \mathcal{B}}^{2}(M ; \rho)\right) \cap W_{p}^{1}\left(J, L_{p}(M)\right) .
$$

The map $\left(f, u_{0}\right) \mapsto u$ is linear and continuous.

Equivalently: Set $A:=\mathcal{A} \mid W_{p, \mathcal{B}}^{2}(M ; \rho)$. Then $-A$ generates a strongly continuous analytic semigroup on $L_{p}(M)$ and has the property of maximal regularity.

This is a particular instance of Theorem 5.2 and its corollary, both of which apply to general weighted spaces, that is, to $\lambda \neq 0$ as well.

We should like to point out that we impose minimal regularity requirements on $a$ (within the framework of continuous coefficients). This allows to use Theorems 1.1 and 1.2 (and the more general results below) as a basis for the study of quasilinear equations along well-established lines (e.g., [1], 3]). For the sake of brevity we do not give details in this paper.

It should also be noted that only the behavior of $\rho$ near zero and infinity is of importance. In other words, if $\tilde{\rho} \in C^{\infty}(M,(0, \infty))$ satisfies $\tilde{\rho} \sim \rho$, that is, $\rho / c \leq \tilde{\rho} \leq c \rho$ for some $c \geq 1$, then Theorem 1.2 remains valid with $\rho$ replaced by $\tilde{\rho}$. In particular, $W_{p, \mathcal{B}}^{2}(M ; \tilde{\rho})$ equals $W_{p, \mathcal{B}}^{2}(M ; \rho)$ except for equivalent norms. 
Now we illustrate the strength of our results by means of relatively simple examples. For this we assume that $\Omega$ is a smooth open subset of $\mathbb{R}^{m}$ with a compact smooth boundary, that is, $\bar{\Omega}$ is a smooth $m$-dimensional submanifold of $\mathbb{R}^{m}$. We also assume that

$\boldsymbol{\Gamma}$ is a finite family of compact connected smooth submanifolds $\Gamma$ of $\mathbb{R}^{m}$ without boundary and dimension $\ell_{\Gamma} \leq m-1$ such that the following applies:

$$
\begin{aligned}
& \text { if } \ell_{\Gamma}=m-1 \text { and } \Gamma \cap \partial \Omega \neq \emptyset \text {, then } \Gamma \subset \partial \Omega \text {. } \\
& \text { if } 1 \leq \ell_{\Gamma} \leq m-2 \text {, then } \Gamma \subset \Omega \text {. }
\end{aligned}
$$

Then $M:=\bar{\Omega} \backslash \bigcup\{\Gamma ; \Gamma \in \Gamma\}$, endowed with the Euclidean metric, is an $m$-dimensional Riemannian submanifold of $\mathbb{R}^{m}$ whose boundary $\partial M$ equals $\partial \Omega \backslash \bigcup\{\Gamma ; \Gamma \in \Gamma\}$.

For each $\Gamma \in \boldsymbol{\Gamma}$ and $x \in M$ we denote by $\delta_{\Gamma}(x)$ the (Euclidean) distance from $x$ to $\Gamma$. Then $\delta_{\Gamma}$ is, sufficiently close to $\Gamma$, a well-defined strictly positive smooth function. If $M$ contains a neighborhood of infinity in $\mathbb{R}^{m}$, that is, if $\Omega$ is an exterior domain, then we put $\delta_{\infty}(x):=|x|$ with the Euclidean norm $|\cdot|$ in $\mathbb{R}^{m}$. We also fix $\alpha_{\Gamma} \geq 1$ and $\alpha_{\infty} \in(-\infty, 0)$. Then we choose a function $\rho \in C^{\infty}(M,(0, \infty))$ satisfying $\rho \sim \delta_{\Gamma}^{\alpha_{\Gamma}}$ near $\Gamma \in \Gamma, \quad \rho \sim \delta_{\infty}^{\alpha_{\infty}}$ near infinity if $\Omega$ is an exterior domain, and $\rho \sim \mathbf{1}$ away from the 'singularity set' $\mathcal{S}(M):=\bigcup\{\Gamma ; \Gamma \in \Gamma\}$ and infinity. Then $M$ is a singular Riemannian manifold characterized by the singularity function $\rho$. Indeed, see Examples 4.1, each $\Gamma \in \boldsymbol{\Gamma}$ is an $\left(\alpha_{\Gamma}, \ell_{\Gamma}\right)$-wedge and $\{x \in M ;|x|>R\}$ is for sufficiently large $R>1$ diffeomorphic to an infinite $\alpha_{\infty}$-cusp (over $S^{m-1}$ in $\mathbb{R}^{m+1}$ ) if $\Omega$ is an exterior domain. Thus Theorem 1.2 applies to this situation.

Next we consider some particularly simple subcases which have been treated before in the literature.

(a) Suppose $\Omega$ is bounded and $\mathcal{S}(M)=\partial \Omega$. Thus $\rho \sim \delta^{\alpha}$ for some $\alpha \geq 1$, where $\delta$ is the distance to $\partial \Omega$. In this situation it is shown by V. Vespri 34 that $A$ generates an analytic semigroup on $L_{p}(\Omega)=L_{p}(M)$. Recently, S. Fornaro, G. Metafune, and D. Pallara [19] have given a new proof for this generation theorem.

(b) Let $\Omega$ be bounded and $\ell_{\Gamma}=0$ for each $\Gamma \in \Gamma$. Then $\mathcal{S}(M)$ consists of finitely many one-point sets $\left\{x_{0}\right\}, \ldots,\left\{x_{k}\right\}$ lying either in $\Omega$ or on $\partial \Omega$. We set $\delta_{j}(x):=\left|x-x_{j}\right|$ for $0 \leq j \leq k$ and $x \in M=\bar{\Omega} \backslash \bigcup_{j=0}^{k}\left\{x_{j}\right\}$. Assume $\alpha_{j} \geq 1$ for $0 \leq j \leq k$. Then Theorem 1.2 implies that, given any $\rho \in C^{\infty}(M,(0, \infty))$ satisfying $\rho \sim \delta_{j}^{\alpha_{j}}$ near $x_{j}$ and $\rho \sim 1$ otherwise, $-A=-\mathcal{A} \mid W_{p, \mathcal{B}}^{2}(M ; \rho)$ generates a strongly continuous analytic semigroup on $L_{p}(M)=L_{p}(\Omega)$ and has the property of maximal regularity.

The only paper known to the author treating the problem of semigroup generation by parabolic equations with strong degeneracies at isolated points is the recent publication of G. Fragnelli, G. Ruiz Goldstein, J.A. Goldstein, and S. Romanelli [20]. These authors consider the case where $\Omega=(0,1)$ and $\mathcal{S}(M)=\left\{x_{0}\right\} \subset \Omega$ and show that $-A$ generates an analytic semigroup on $L_{2}(\Omega)$. 
In none of the above papers it is shown that the maximal regularity property prevails. Furthermore, the proofs given there depend significantly on the fact that second order equations are being considered. In contrast, our approach does not depend on the particular structure of the problem but applies equally well to systems and higher order equations (cf. H. Amann [7]).

Observe that the preceding examples show that a given Riemannian manifold can possess uncountably many non-equivalent singular structures. This is related to and sheds new light on the non-uniqueness results observed by M.A. Pozio, F. Punzo, and A. Tesei [30. Thus, besides being rather general and widely applicable, our approach to highly degenerate parabolic problems via Riemannian manifolds leads to a deeper understanding of such problems as well.

In the next section we give the precise definition of a uniformly regular Riemannian manifold. Then we formulate our main result, Theorem 3.1 , in the setting of second order equations and trace it back to the much more general propositions in [7. Note that, besides allowing lower order terms, we prove an optimal regularity theorem in the presence of nonhomogeneous boundary conditions. In addition, we show that we get classical solutions if we impose slightly stronger regularity assumptions on the data.

Singular Riemannian manifolds are precisely defined in Section 4 and basic examples are presented. Furthermore, weighted function spaces are introduced and their interrelation with non-weighted Sobolev-Slobodeckii spaces on uniformly regular manifolds is established.

Section 5 contains our main theorem for second order degenerate parabolic problems involving lower order terms and nonhomogeneous boundary conditions. We attract the reader's attention to Theorem 5.2 where it is shown that problems with homogeneous boundary conditions give rise to generators of analytic semigroups possessing the property of maximal regularity in general weighted spaces $L_{p}^{\lambda}(M ; \rho)$ for any $\lambda \in \mathbb{R}$. This generalizes results by V. Barbu, A. Favini, and S. Romanelli [15, for example, where the case $M=\Omega$, with $\Omega$ a bounded domain on $\mathbb{R}^{m}, \mathcal{S}(M)=\partial \Omega$, and $\lambda=-1$ is considered (see also [20]).

For the reader's convenience there is included an appendix in which some basic facts on tensor bundles over Riemannian manifolds are listed.

\section{Function Spaces and Uniformly Regular Manifolds}

By a manifold we always mean a smooth, that is, $C^{\infty}$ manifold with (possibly empty) boundary such that its underlying topological space is separable and metrizable. Thus, in the context of manifolds, we work in the smooth category. A manifold does not need to be connected, but all connected components are of the same dimension.

Let $M=(M, g)$ be a Riemannian manifold with boundary $\partial M$ and volume measure $d v$. The metric $g$ on $T M$ gives rise to a vector bundle metric on the tensor bundle $V_{\tau}^{\sigma}:=T_{\tau}^{\sigma} M$ for $\sigma, \tau \in \mathbb{N}$, which we denote by $g_{\sigma}^{\tau}$ (see 
the appendix for more details). In particular, $g_{1}^{0}=g$ and $g_{0}^{1}=g^{*}$, the adjoint (or contravariant) metric on the cotangent bundle $T^{*} M$.

For $k \in \mathbb{N}$ the vector space of all $(\sigma, \tau)$-tensor fields of class $C^{k}$, that is, of all $C^{k}$ sections of $V_{\tau}^{\sigma}$, is denoted by $C^{k}\left(V_{\tau}^{\sigma}\right)$. The Levi-Civita covariant derivative, $\nabla$, satisfies $\nabla^{k} a \in C\left(V_{\tau+k}^{\sigma}\right)$ for $a \in C^{k}\left(V_{\tau}^{\sigma}\right)$, where $\nabla^{0} a:=a$. We write $\mathcal{D}\left(V_{\tau}^{\sigma}\right)$ for the space of all smooth sections with compact support in $M$ (which may meet the boundary). As usual, $C^{k}(M)$ stands for $C^{k}\left(V_{0}^{0}\right)$, etc.

We fix $\sigma$ and $\tau$ and set $V:=V_{\tau}^{\sigma}$. Then, given $k \in \mathbb{N}$, we denote by $W_{p}^{k}(V)$ the Sobolev space of order $k$, defined to be the completion of $\mathcal{D}(V)$ in $L_{1, \text { loc }}(V)=L_{1, \text { loc }}(V, d v)$ with respect to the norm

$$
u \mapsto\left(\sum_{j=0}^{k}\left\|\left|\nabla^{j} u\right|_{g_{\sigma}^{\tau+j}}\right\|_{L_{p}(V)}^{p}\right)^{1 / p} .
$$

Thus $W_{p}^{0}(V)=L_{p}(V)$.

We also need fractional order Sobolev spaces, namely the Slobodeckii spaces $W_{p}^{s}(V)$, for $s \in \mathbb{R}^{+} \backslash \mathbb{N}$. If $k<s<k+1$ with $k \in \mathbb{N}$, then

$$
W_{p}^{s}(V):=\left(W_{p}^{k}(V), W_{p}^{k+1}(V)\right)_{s-k, p},
$$

which is the interpolation space between $W_{p}^{k}(V)$ and $W_{p}^{k+1}(V)$ obtained by means of the real interpolation method with exponent $s-k$ and integrability parameter $p$.

We denote by $B(V)$ the space of all bounded sections of $V$. It is a Banach space with the norm $u \mapsto\|u\|_{\infty}:=\||u|\|_{\infty}$, where $\|\cdot\|_{\infty}$ is the maximum norm. Moreover, $B C(V):=B(V) \cap C(V)$ is a closed linear subspace thereof. For $k \in \mathbb{N}$ we write $B C^{k}(V)$ for the linear subspace of $C^{k}(V)$ consisting of all $u$ satisfying $\nabla^{j} u \in B\left(V_{\tau+j}^{\sigma}\right)$ for $0 \leq j \leq k$. It is a Banach space with the obvious norm. Moreover, $B C^{\infty}(V):=\bigcap_{k} B C^{k}(V)$ and $b c^{k}(V)$ is the closure of $B C^{\infty}(V)$ in $B C^{k}(V)$. Now we define Besov-Hölder spaces $B_{\infty}^{s}(V)$ for $s>0$ by

$$
B_{\infty}^{s}(V):=\left\{\begin{array}{lr}
\left(b c^{k}(V), b c^{k+1}(V)\right)_{s-k, \infty}, & k<s<k+1, \\
\left(b c^{k}(V), b c^{k+2}(V)\right)_{1 / 2, \infty}, & s=k+1,
\end{array}\right.
$$

where $k \in \mathbb{N}$.

Besides these isotropic spaces we also need anisotropic versions adapted to parabolic problems. Anisotropic Sobolev-Slobodeckii spaces are introduced for $s \in \mathbb{R}^{+}=[0, \infty)$ by

$$
W_{p}^{(s, s / 2)}(V \times J):=L_{p}\left(J, W_{p}^{s}(V)\right) \cap W_{p}^{s / 2}\left(J, L_{p}(V)\right) .
$$

The second space on the right is a standard Sobolev-Slobodeckii space of Banach space valued distributions on $\stackrel{\circ}{J}$. Of course, $W_{p}^{(0,0)}(V \times J)$ is naturally identified with $L_{p}(V \times J)=L_{p}(V \times J, d v d t)$.

Analogously, we define anisotropic Besov-Hölder spaces for $s>0$ by

$$
B_{\infty}^{(s, s / 2)}(V \times J):=B\left(J, B_{\infty}^{s}(V)\right) \cap B_{\infty}^{s / 2}(J, B(V)) .
$$


Here the second space on the right is a standard Hölder space $C^{s / 2}(J, B(V))$ if $s \notin 2 \mathbb{N}$, and a Zygmund space for $s \in 2 \mathbb{N}$, of Banach space valued functions on $J$ (see A. Lunardi [28, for example). For $k \in \mathbb{N}^{\times}:=\mathbb{N} \backslash\{0\}$ we put

$$
B C^{(k, k / 2)}(V \times J):=C\left(J, B C^{k}(V)\right) \cap C^{k / 2}(J, B(V)),
$$

recalling that $J$ is compact.

Although Sobolev-Slobodeckii spaces, respectively Besov-Hölder spaces, are well-defined for each $s \in \mathbb{R}^{+}$, respectively $s>0$, they are not too useful on general Riemannian manifolds since, for example, the fundamental Sobolev type embedding theorems may not hold in general. Even more importantly, there may be no characterization by local coordinates. For this reason we restrict ourselves to the class of uniformly regular Riemannian manifolds. Loosely speaking, $M$ is a uniformly regular Riemannian manifold if its differentiable structure is induced by an atlas $\mathfrak{K}$ of finite multiplicity whose coordinate patches are all of comparable size, such that $\mathfrak{K}$ can be uniformly shrunk to an atlas for $M$, and the family of all charts in $\mathfrak{K}$ which intersect $\partial M$ induces an atlas of the same type for $\partial M$. In particular, $\partial M=(\partial M, \dot{g})$, where $\dot{g}$ is the Riemannian metric induced by $g$ on $\partial M$, is a uniformly regular $(m-1)$-dimensional Riemannian manifold if $M$ is uniformly regular (see Example 4.1(b)).

For the precise definition of a uniformly regular Riemannian manifold we introduce some notation and conventions. By $c$ we denote constants $\geq 1$ whose numerical value may vary from occurrence to occurrence; but $c$ is always independent of the free variables in a given formula, unless a dependence is explicitly indicated.

We denote by $\mathbb{H}^{m}$ the closed right half-space $\mathbb{R}^{+} \times \mathbb{R}^{m-1}$ in $\mathbb{R}^{m}$, where $\mathbb{R}^{0}=\{0\}$. The Euclidean metric on $\mathbb{R}^{m},\left(d x^{1}\right)^{2}+\cdots+\left(d x^{m}\right)^{2}$, is denoted by $g_{m}$. The same symbol is used for its restriction to an open subset $U$ of $\mathbb{R}^{m}$ or $\mathbb{H}^{m}$, that is, for $\iota^{*} g_{m}$, where $\iota: U \hookrightarrow \mathbb{R}^{m}$ is the natural embedding. Here and below, we employ standard definitions of pull-back and push-forward operations.

On the space of all nonnegative functions, defined on some nonempty set whose specific form will be clear in any given situation, we introduce an equivalence relation $\sim$ by setting $f \sim g$ iff there exists $c \geq 1$ such that $f / c \leq g \leq c f$. Inequalities between vector bundle metrics have to be understood in the sense of quadratic forms. By 1 we denote the constant function $s \mapsto 1$, whose domain will always be clear from the context.

We set $Q:=(-1,1) \subset \mathbb{R}$. If $\kappa$ is a local chart for an $m$-dimensional manifold $M$, then we write $U_{\kappa}$ for the corresponding coordinate patch $\operatorname{dom}(\kappa)$.

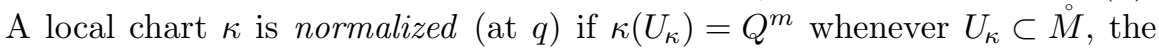
interior of $M$, whereas $\kappa\left(U_{\kappa}\right)=Q^{m} \cap \mathbb{H}^{m}$ if $U_{\kappa} \cap \partial M \neq \emptyset($ and $\kappa(q)=0)$. We put $Q_{\kappa}^{m}:=\kappa\left(U_{\kappa}\right)$ if $\kappa$ is normalized.

An atlas $\mathfrak{K}$ for $M$ has finite multiplicity if there exists $k \in \mathbb{N}$ such that any intersection of more than $k$ coordinate patches is empty. In this case

$$
\mathfrak{N}(\kappa):=\left\{\tilde{\kappa} \in \mathfrak{K} ; U_{\tilde{\kappa}} \cap U_{\kappa} \neq \emptyset\right\}
$$


has cardinality $\leq k$ for each $\kappa \in \mathfrak{K}$. An atlas is uniformly shrinkable if it consists of normalized charts and there exists $r \in(0,1)$ such that the family $\left\{\kappa^{-1}\left(r Q_{\kappa}^{m}\right) ; \kappa \in \mathfrak{K}\right\}$ is a cover of $M$. We put $\mathfrak{K}_{S}:=\left\{\kappa \in \mathfrak{K} ; U_{\kappa} \cap S \neq \emptyset\right\}$ for any nonempty subset $S$ of $M$.

Given an open subset $X$ of $\mathbb{R}^{m}$ or $\mathbb{H}^{m}$ and a Banach space $\mathcal{X}$, we write $\|\cdot\|_{k, \infty}$ for the usual norm of $B C^{k}(X, \mathcal{X})$, the Banach space of all $u \in C^{k}(X, \mathcal{X})$ such that $\left|\partial^{\alpha} u\right|_{\mathcal{X}}$ is uniformly bounded for $\alpha \in \mathbb{N}^{m}$ of length at most $k$.

An atlas $\mathfrak{K}$ for $M$ is uniformly regular if

(i) $\quad \mathfrak{K}$ is uniformly shrinkable and has finite multiplicity.

(ii) $\quad\left\|\tilde{\kappa} \circ \kappa^{-1}\right\|_{k, \infty} \leq c(k), \quad \kappa, \tilde{\kappa} \in \mathfrak{K}, \quad k \in \mathbb{N}$.

In (ii) and in similar situations it is understood that only $\kappa, \tilde{\kappa} \in \mathfrak{K}$ with $U_{\kappa} \cap U_{\tilde{\kappa}} \neq \emptyset$ are being considered. Two uniformly regular atlases $\mathfrak{K}$ and $\tilde{\mathfrak{K}}$ are equivalent, $\mathfrak{K} \approx \tilde{\mathfrak{K}}$, if

$$
\begin{aligned}
& \operatorname{card}\left\{\tilde{\kappa} \in \tilde{\mathfrak{K}} ; U_{\tilde{\kappa}} \cap U_{\kappa} \neq \emptyset\right\} \leq c, \quad \kappa \in \mathfrak{K} ; \\
& \left\|\tilde{\kappa} \circ \kappa^{-1}\right\|_{k, \infty}+\left\|\kappa \circ \tilde{\kappa}^{-1}\right\|_{k, \infty} \leq c(k), \quad \kappa \in \mathfrak{K}, \quad \tilde{\kappa} \in \tilde{\mathfrak{K}}, \quad k \in \mathbb{N} .
\end{aligned}
$$

A uniformly regular structure is a maximal family of equivalent uniformly regular atlases. A uniformly regular manifold is a manifold endowed with a uniformly regular structure. Clearly, on such a manifold all local charts, atlases, etc. under consideration belong to its uniformly regular structure. An $m$-dimensional Riemannian manifold $(M, g)$ is a uniformly regular Riemannian manifold if

(i) $\quad M$ is uniformly regular;

(ii) $\quad \kappa_{*} g \sim g_{m}, \quad \kappa \in \mathfrak{K}$;

(iii) $\quad\left\|\kappa_{*} g\right\|_{k, \infty} \leq c(k), \quad \kappa \in \mathfrak{K}, \quad k \in \mathbb{N}$,

for some uniformly regular atlas $\mathfrak{K}$ for $M$.

Let $M$ be a uniformly regular Riemannian manifold. Then the SobolevSlobodeckii and Besov-Hölder space scales possess all the properties known to hold in the case of the $m$-dimensional Euclidean space or half-space. In other words, there are embedding, interpolation, and trace theorems for $W_{p}^{s}(M)$ and $W_{p}^{(s, s / 2)}(M \times J)$ which are completely analogous to the corresponding theorems for the classical Sobolev-Slobodeckii spaces. In particular, the anisotropic Sobolev-Morrey type embedding theorem

$$
W_{p}^{(s, s / 2)}(V \times J) \hookrightarrow B_{\infty}^{(t, t / 2)}(V \times J), \quad s+(m+2) / p>t>0,
$$

is valid. In addition, $W_{p}^{s}(V)$ and $B_{\infty}^{s}(V)$ can be characterized by means of local coordinates, similarly as in the case of compact manifolds.

The spaces $B C^{k}(V)$ and $B C^{(k, k / 2)}(V)$ do not belong to either one of these scales. However, they can be arbitrarily well approximated by BesovHölder spaces. In fact, given $k \in \mathbb{N}^{\times}$,

$$
B_{\infty}^{\left(s_{1}, s_{1} / 2\right)}(V \times J) \hookrightarrow B C^{(k, k / 2)}(V \times J) \hookrightarrow B_{\infty}^{\left(s_{0}, s_{0} / 2\right)}(V \times J)
$$


for $0<s_{0}<k<s_{1}$. Note that this implies a corresponding assertion for the isotropic spaces $B C^{k}(V)$ and $B_{\infty}^{s}(V)$, since $B C^{k}(V)$ is naturally identified with the closed linear subspace of $B C^{(k, k / 2)}(V)$ of all 'time-independent' functions therein, etc.

Proofs, further results, references to related research, and many more details - in particular spaces of sections of general uniformly regular vector bundles over $M$ - are found in the earlier work [6], 5] of the author (also see [7, [8], as well as [4]).

\section{Parabolic Problems on Uniformly Regular Riemannian Manifolds}

Let $M=(M, g)$ be a uniformly regular Riemannian manifold. We consider parabolic initial boundary value problems of the form

$$
\partial u+\mathcal{A} u=f \text { on } M_{T}, \quad \mathcal{B} u=h \text { on } \partial M_{T}, \quad u=u_{0} \text { on } M_{0} .
$$

In order to reduce the technical apparatus to a minimum we restrict ourselves to the important class of second order divergence form problems. Thus we fix $\delta \in C(\partial M,\{0,1\})$ and set $\partial_{j} M:=\delta^{-1}(j)$ for $j \in\{0,1\}$. Then $\partial M=\partial_{0} M \cup \partial_{1} M$ and $\partial_{0} M \cap \partial_{1} M=\emptyset$. We assume that $(\mathcal{A}, \mathcal{B})$ is of the form

$$
\mathcal{A} u:=-\operatorname{div}(a \cdot \operatorname{grad} u)+(\vec{a} \mid \operatorname{grad} u)+a_{0} u
$$

and

$$
\mathcal{B} u:= \begin{cases}\mathcal{B}_{0} u & \text { on } \partial_{0} M_{T}, \\ \mathcal{B}_{1} u & \text { on } \partial_{1} M_{T},\end{cases}
$$

where

$$
\mathcal{B}_{0} u:=\gamma u, \quad \mathcal{B}_{1} u:=(\nu \mid \gamma(a \cdot \operatorname{grad} u))+b_{0} \gamma u .
$$

Here $(\cdot \mid \cdot)=(\cdot \mid \cdot)_{g}:=g(\cdot, \cdot), \quad \nu$ is the (inward pointing) unit normal on $\partial M$, $\gamma$ the trace map for $\partial M$, and - denotes complete contraction (see the appendix). More precisely, $\mathcal{B}_{0} u=(\gamma u) \mid \partial_{0} M$, etc. We suppose $a \in C^{1}\left(T_{1}^{1} M \times J\right)$, $\vec{a}$ is a time-dependent vector field, $a_{0}$ a function on $M_{T}$, and $b_{0}$ one on $\partial_{1} M_{T}$. In local coordinates,

$$
\mathcal{A} u=-\frac{1}{\sqrt{g}} \partial_{i}\left(\sqrt{g} a_{j}^{i} g^{j k} \partial_{k} u\right)+a^{i} \partial_{i} u+a_{0} u .
$$

Hence $\mathcal{B}$ is the Dirichlet boundary operator on $\partial_{0} M$ and the Neumann or a Robin boundary operator on $\partial_{1} M$. Note that either $\partial_{0} M$ or $\partial_{1} M$ may be empty. We also allow $M$ to be a manifold without boundary. In this case it is understood throughout the whole paper that all statements, assumptions, and formulas referring explicitly or implicitly to $\partial M$ are to be unconsidered. For example, problem (3.1) reduces to the Cauchy problem

$$
\partial u+\mathcal{A} u=f \text { on } M_{T}, \quad u=u_{0} \text { on } M_{0}
$$

if $\partial M=\emptyset$. 
A function $u$ satisfying (3.1) is a strong $L_{p}$ solution if it belongs to $W_{p}^{(2,1)}\left(M_{T}\right)$, and a classical solution if it is a member of $B C^{(2,1)}\left(M_{T}\right)$.

The differential operator $\mathcal{A}$ is uniformly strongly elliptic on $M_{T}$ if $a(\cdot, t)$ is symmetric and uniformly positive definite, uniformly with respect to $t \in J$. Clearly, the latter means that there exists a constant $\varepsilon>0$ such that

$$
(a(q, t) \cdot X \mid X)_{g(q)} \geq \varepsilon|X|_{g(q)}^{2}, \quad X \in T_{q} M, \quad q \in M, \quad t \in J .
$$

For a concise formulation of the main result we introduce for $s \geq 0$ the boundary data spaces

$$
\begin{aligned}
& W_{p}^{(s+2-\vec{\delta}-1 / p)(1,1 / 2)}\left(\partial M_{T}\right) \\
& \quad:=W_{p}^{(s+2-1 / p)(1,1 / 2)}\left(\partial_{0} M_{T}\right) \times W_{p}^{(s+1-1 / p)(1,1 / 2)}\left(\partial_{1} M_{T}\right),
\end{aligned}
$$

whose general point is written $h=\left(h_{0}, h_{1}\right)$. Obvious interpretations apply if either $\partial_{0} M$ or $\partial_{1} M$ is empty. The total data spaces are then

$$
\begin{aligned}
& \mathcal{W}_{p}^{(s+2,(s+2) / 2)}\left(M_{T}\right) \\
& \quad:=W_{p}^{(s, s / 2)}\left(M_{T}\right) \times W_{p}^{(s+2-\vec{\delta}-1 / p)(1,1 / 2)}\left(\partial M_{T}\right) \times W_{p}^{s+2-2 / p}\left(M_{0}\right)
\end{aligned}
$$

for $s \geq 0$.

Given Banach spaces $E$ and $F$, we denote by $\mathcal{L}(E, F)$ the Banach space of bounded linear operators from $E$ into $F$. We write $\mathcal{L}$ is $(E, F)$ for the subset of all bijections in $\mathcal{L}(E, F)$. Banach's homomorphism theorem guarantees that $A^{-1} \in \mathcal{L}(F, E)$ if $A \in \mathcal{L}$ is $(E, F)$.

Now we can formulate the main existence and uniqueness theorem for problem (3.1).

Theorem 3.1. Let $M$ be a uniformly regular Riemannian manifold and let $p \notin\{3 / 2,3\}$. Suppose

$$
\begin{gathered}
a \in B C^{(1,1 / 2)}\left(T_{1}^{1} M \times J\right), \quad \vec{a} \in L_{\infty}(T M \times J), \\
a_{0} \in L_{\infty}\left(M_{T}\right), \quad b_{0} \in B C^{(1,1 / 2)}\left(\partial_{1} M_{T}\right),
\end{gathered}
$$

and $\mathcal{A}$ is uniformly strongly elliptic. Denote by $\mathcal{W}_{p, \mathrm{cc}}^{(2,1)}\left(M_{T}\right)$ the vector space of all $\left(f, h, u_{0}\right) \in \mathcal{W}_{p}^{(2,1)}\left(M_{T}\right)$ satisfying the compatibility conditions of order zero:

$$
\begin{aligned}
\gamma u_{0} & =h_{0}(\cdot, 0) & \text { on } \partial_{0} M & & \text { if } & 3 / 2<p<3, \\
\mathcal{B}(\cdot, 0) u_{0} & =h(\cdot, 0) & \text { on } \partial M & \text { if } & p & >3 .
\end{aligned}
$$

Then $\mathcal{W}_{p, \mathrm{cc}}^{(2,1)}\left(M_{T}\right)$ is closed in $\mathcal{W}_{p}^{(2,1)}\left(M_{T}\right)$ and

$$
\left(\partial+\mathcal{A}, \mathcal{B}, \gamma_{0}\right) \in \mathcal{L} \operatorname{is}\left(W_{p}^{(2,1)}\left(M_{T}\right), \mathcal{W}_{p, \mathrm{cc}}^{(2,1)}\left(M_{T}\right)\right) .
$$

Supplement. Suppose $0<s<\bar{s}<1+3 / p$ with $s \neq 3 / p$ and

$$
\begin{gathered}
a \in B_{\infty}^{(1+\bar{s})(1,1 / 2)}\left(T_{1}^{1} M \times J\right), \quad \vec{a} \in B_{\infty}^{(\bar{s}, \bar{s} / 2)}(T M \times J), \\
a_{0} \in B_{\infty}^{(\bar{s}, \bar{s} / 2)}\left(M_{T}\right), \quad b_{0} \in B_{\infty}^{(1+\bar{s})(1,1 / 2)}\left(\partial_{1} M_{T}\right) .
\end{gathered}
$$


Let $\mathcal{W}_{p, \mathrm{cc}}^{(s+2)(1,1 / 2)}\left(M_{T}\right)$ be the linear subspace of $\mathcal{W}_{p}^{(s+2)(1,1 / 2)}\left(M_{T}\right)$ of all $\left(f, h, u_{0}\right)$ satisfying, in addition to (3.4), the first order compatibility condition

$$
\partial h_{0}(\cdot, 0)+\gamma \mathcal{A}(\cdot, 0) u_{0}=\gamma f(\cdot, 0) \text { on } \partial_{0} M \quad \text { if } s>2 / p .
$$

Then $\mathcal{W}_{p, \mathrm{cc}}^{(s+2)(1,1 / 2)}\left(M_{T}\right)$ is closed and

$$
\left(\partial+\mathcal{A}, \mathcal{B}, \gamma_{0}\right) \in \mathcal{L} \operatorname{is}\left(W_{p}^{(s+2)(1,1 / 2)}\left(M_{T}\right), \mathcal{W}_{p, \mathrm{cc}}^{(s+2)(1,1 / 2)}\left(M_{T}\right)\right) .
$$

Proof. We set $a_{2}:=-a^{\sharp}$ and $a_{1}:=\vec{a}-\operatorname{div}\left(a^{\sharp}\right)$, using the notations of the appendix. Then we get from A.12

$$
\mathcal{A}=a_{2} \cdot \nabla^{2}+a_{1} \cdot \nabla+a_{0} .
$$

We let $\nu_{b}$ be the unit conormal vector field $g_{b} \nu$ on $\partial M$ and set $b_{1}:=\nu_{b} \cdot \gamma a^{\sharp}$. Then

$$
\mathcal{B}_{1}=b_{1} \cdot \gamma \nabla+b_{0} \gamma
$$

By means of the characterization of $B C^{k}\left(T^{*} M\right)$ by local coordinates referred to in the preceding section one verifies

$$
\nu_{b} \in B C^{\infty}\left(T^{*} M\right) .
$$

Let (3.3) be satisfied. Then it is obvious that

$$
a_{2} \in B C^{(1,1 / 2)}\left(T_{0}^{2} M \times J\right), \quad a_{1} \in L_{\infty}(T M \times J), \quad a_{0} \in L_{\infty}\left(M_{T}\right) .
$$

Furthermore, (3.10) implies

$$
b_{1} \in B C^{(1,1 / 2)}\left((T M) \mid \partial_{1} M \times J\right) .
$$

If (3.6) applies, then

$$
\begin{gathered}
a_{2} \in B_{\infty}^{(1+\bar{s})(1,1 / 2)}\left(T_{0}^{2} M \times J\right), \quad a_{1} \in B_{\infty}^{(\bar{s}, \bar{s} / 2)}(T M \times J), \\
a_{0} \in B_{\infty}^{(\bar{s}, \bar{s} / 2)}\left(M_{T}\right),
\end{gathered}
$$

and, once more by (3.10) and the point-wise multiplier result [5, Theorem 14.3],

$$
b_{1} \in B_{\infty}^{(1+\bar{s})(1,1 / 2)}\left((T M)_{\mid \partial_{1} M} \times J\right) .
$$

This shows that $(\mathcal{A}, \mathcal{B})$ satisfies in either case the regularity assumptions of the main theorem of [7]. Since the uniform strong ellipticity of $\mathcal{A}$ implies that $(\partial+\mathcal{A}, \mathcal{B})$ is a uniformly strongly parabolic boundary value problem the assertion is a very particular consequence of the latter theorem.

Corollary 3.2. Let (3.3) be satisfied. Then the initial boundary value problem (3.1) has for each $\left(f, h, u_{0}\right) \in \mathcal{W}_{p, \mathrm{cc}}^{(2,1)}\left(M_{T}\right)$ a unique strong $L_{p}$ solution $u$ on $M_{T}$. Suppose $(m+2) / p<s<1+3 / p$ with $s \neq 3 / p$, (3.6) applies, and $\left(f, h, u_{0}\right) \in \mathcal{W}_{p, \mathrm{cc}}^{(s+2)(1,1 / 2)}\left(M_{T}\right)$, then $u$ is a classical solution.

Proof. The first assertion is clear and the second one follows from (2.6). 
Remarks 3.3. (a) If $\delta=0$ (Dirichlet boundary value problem), then $p=3$ is admissible as well. If $\delta=\mathbf{1}$ (Neumann or Robin boundary conditions), then $p=3 / 2$ can be admitted also. Similarly, (3.7) is vacuous if $\delta=1$.

(b) If all data are smooth and the compatibility conditions of all orders are satisfied, then $u$ is a smooth solution on $M_{T}$.

(c) We refer to [7] for higher order problems and operators acting on sections of general uniformly regular vector bundles over $M$.

(d) Theorem 3.1 is the basis for establishing results on the existence, uniqueness, and continuous dependence on the data of solutions of quasilinear parabolic problems of the form

$$
\partial u+\mathcal{A}(u) u=F(u) \text { on } M_{T}, \quad \mathcal{B}(u) u=H(u) \text { on } \partial M_{T}, \quad u=u_{0} \text { on } M_{0} .
$$

Such results are obtained by (more or less obvious) modifications of the proofs in [3. This is to be carried out somewhere else.

Of course, Theorem 3.1 applies in particular to autonomous problems. To simplify the presentation we restrict ourselves to the setting of strong $L_{p}$ solutions. Then (3.3) reduces to

$$
\begin{aligned}
& a \in B C^{1}\left(T_{1}^{1} M\right), \quad \vec{a} \in L_{\infty}(T M), \\
& a_{0} \in L_{\infty}(M), \quad b_{0} \in B C^{1}\left((T M)_{\mid \partial_{1} M}\right) \text {. }
\end{aligned}
$$

Of particular importance is the case of homogeneous boundary value problems.

Theorem 3.1 guarantees $\mathcal{A} \in \mathcal{L}\left(W_{p}^{2}(M), L_{p}(M)\right)$. Hence $A$, the restriction of $\mathcal{A}$ to $W_{p, \mathcal{B}}^{2}(M)$, is a well-defined element of $\mathcal{L}\left(W_{p, \mathcal{B}}^{2}(M), L_{p}(M)\right)$. Moreover, $A$ is closed (cf. [1, Lemma I.1.1.2]) and densely defined (since $\mathcal{D}(\stackrel{\circ}{)})$ is a subset of $W_{p, \mathcal{B}}^{2}(M)$ and $\mathcal{D}(\dot{M})$ is dense in $\left.L_{p}(M)\right)$. By means of $A$ we can reformulate the autonomous homogeneous initial boundary value problem (1.2) as the evolution equation (1.6). This is made precise by the next theorem for which we rely on semigroup theory and maximal regularity (see H. Amann [1, Chapter III] and [2, R. Denk, M. Hieber, and J. Prüss [17], or P.Ch. Kunstmann and L. Weis [26], for example, for information on these concepts).

Theorem 3.4. Let $M$ be a uniformly regular Riemannian manifold and let $p \notin\{3 / 2,3\}$. Suppose $\mathcal{A}$ is autonomous, uniformly strongly elliptic, and conditions (3.11) are satisfied. Then $-A$ generates a strongly continuous analytic semigroup on $L_{p}(M)$ and has the property of maximal regularity, that is to say, $\left(\partial+A, \gamma_{0}\right)$ belongs to

$$
\mathcal{L} \operatorname{is}\left(L_{p}\left(J, W_{p, \mathcal{B}}^{2}(M)\right) \cap W_{p}^{1}\left(J, L_{p}(M)\right), L_{p}\left(M_{T}\right) \times W_{p, \mathcal{B}}^{2-2 / p}(M)\right) .
$$

Proof. In the present setting $\mathcal{W}_{p, \mathrm{cc}}^{(2,1)}\left(M_{T}\right)=L_{p}\left(M_{T}\right) \times W_{p, \mathcal{B}}^{2-2 / p}(M)$. Hence (3.12) is a reformulation of (3.5). Now the semigroup assertion follows from a result of G. Dore [18]. 
To indicate the power of these theorems we need to know examples of uniformly regular Riemannian manifolds. This problem is dealt with in 9] where proofs for the following claims are found.

Examples 3.5. (a) Every compact Riemannian manifold is a uniformly regular Riemannian manifold.

(b) An $m$-dimensional Riemannian submanifold of $\mathbb{R}^{m}$ possessing a compact boundary is a uniformly regular Riemannian manifold.

(c) $\mathbb{R}^{m}=\left(\mathbb{R}^{m}, g_{m}\right)$ and $\mathbb{H}^{m}=\left(\mathbb{H}^{m}, g_{m}\right)$ are uniformly regular Riemannian manifolds.

(d) Let $\tilde{M}=(\tilde{M}, \tilde{g})$ be a Riemannian manifold and $\varphi:(M, g) \rightarrow(\tilde{M}, \tilde{g})$ an isometry. Then $M$ is a uniformly regular Riemannian manifold iff $\tilde{M}$ is one.

(e) A Riemannian manifold has bounded geometry if it has no boundary, a positive injectivity radius, and all covariant derivatives of the curvature tensor are bounded. Every complete Riemannian manifold with bounded geometry is a uniformly regular Riemannian manifold.

(f) Suppose $S \subset U \subset M$, where $S$ is closed and $U$ is open in $M$. An atlas $\mathfrak{K}$ for $U$ is uniformly regular on $S$ if (2.3) holds with $\mathfrak{K}$ replaced by $\mathfrak{K}_{S}$. Two uniformly regular atlases $\mathfrak{K}$ and $\tilde{\mathfrak{K}}$ for $U$ on $S$ are equivalent if (2.4) applies to $\mathfrak{K}_{S}$ and $\tilde{\mathfrak{K}}_{S}$. This defines a uniformly regular structure for $U$ on $S$. Then $U$ is uniformly regular on $S$ if it is endowed with a uniformly regular structure on $S$. Lastly, $U$ is a uniformly regular Riemannian manifold on $S$ if (2.5) is satisfied for $U$ and $\mathfrak{K}_{S}$, where $\mathfrak{K}$ is a uniformly regular atlas for $U$ on $S$.

Let $S_{j} \subset U_{j} \subset M$ and suppose $U_{j}$ is a uniformly regular Riemannian manifold on $S_{j}$ for $0 \leq j \leq \ell$. Let $\mathfrak{K}_{j}$ be a uniformly regular atlas for $U_{j}$ on $S_{j}$. Assume

$(\alpha)\left\|\kappa_{i} \circ \kappa_{j}^{-1}\right\|_{k, \infty} \leq c(k), \quad\left(\kappa_{i}, \kappa_{j}\right) \in \mathfrak{K}_{i, S_{i}} \times \mathfrak{K}_{j, S_{j}}, \quad 0 \leq i, j \leq \ell, \quad k \in \mathbb{N} ;$

( $\beta) M=S_{0} \cup \cdots \cup S_{\ell}$.

Then $\mathfrak{K}:=\mathfrak{K}_{0} \cup \cdots \cup \mathfrak{K}_{\ell}$ is a uniformly regular atlas for $M$ and $M$ is a uniformly regular Riemannian manifold. It is obtained by patching together the uniformly regular pieces $U_{j}$ on $S_{j}$.

(g) Assume $d \geq m$ and $B$ is an $(m-1)$-dimensional compact submanifold of $\mathbb{R}^{d-1}$. For a nonempty subinterval $I$ of $(1, \infty)$ and $0 \leq \alpha \leq 1$ we set

$$
F_{\alpha}(I, B):=\left\{\left(t, t^{\alpha} y\right) ; t \in I, y \in B\right\} \subset \mathbb{R} \times \mathbb{R}^{d-1}=\mathbb{R}^{d},
$$

Then $F_{\alpha}(B):=F_{\alpha}((1, \infty), B)$, endowed with the Riemannian metric induced by $\mathbb{R}^{d}$, that is, by $g_{d}$, is an $m$-dimensional Riemannian submanifold of $\mathbb{R}^{d}$ with boundary $F_{\alpha}(\partial B)$, where $F_{\alpha}(\emptyset):=\emptyset$. It is called $\alpha$-funnel in $\mathbb{R}^{d}$. Note that a 0 -funnel is a cylinder and a 1-funnel a (blunt) cone over (the basis) $B$.

Let $F=F_{\alpha}(B)$ be an $\alpha$-funnel in $\mathbb{R}^{d}$ and set $S:=F_{\alpha}([2, \infty), B)$. Then $F$ is an $m$-dimensional uniformly regular Riemannian manifold on $S$.

(h) Suppose $U$ is open in $M$ and $F=F_{\alpha}(B)$ an $m$-dimensional $\alpha$-funnel in $\mathbb{R}^{d}$. Set $F(I):=F_{\alpha}(I, B)$. Assume $\varphi: U \rightarrow F$ is a diffeomorphism such that $S:=\varphi^{-1}(F[2, \infty))$ satisfies 
$(\alpha)(\overline{U \backslash S}) \cap S=\varphi^{-1}(F(\{2\})) ;$

$(\beta) \varphi_{*}(g \mid S) \sim g_{F} \mid F[2, \infty)$.

Then $U$ is a uniformly regular Riemannian manifold on $S$, and $M$ is said to have an $(\alpha, B)$-funnel-like end in $U$ with representation $\varphi$.

(i) Let $U_{0}, \ldots, U_{\ell}$ be open in $M$. Suppose

( $\alpha) U_{i} \cap U_{j}=\emptyset, \quad 1 \leq i<j \leq \ell$;

( $\beta) M$ has an $\left(\alpha_{j}, B_{j}\right)$-funnel-like end in $U_{j}$ with representation $\varphi_{j}$ for $j \geq 1$

$(\gamma) \varphi_{j}\left(U_{0} \cap U_{j}\right)=F_{j}(1,4), \quad j \geq 1$;

( $\delta) S_{0}:=U_{0} \backslash \bigcup_{j=1}^{\ell} \varphi_{j}^{-1}\left(F_{j}(3, \infty)\right)$ is compact.

Then $M$ is a uniformly regular Riemannian manifold, a Riemannian manifold with finitely many funnel-like ends. It is obtained by patching together the uniformly regular pieces $U_{j}$ on $S_{j}$ for $0 \leq j \leq \ell$.

The most elementary situation in which Theorem 3.1 applies is the case in which $M$ is compact. If, notably, $M$ is the closure of a smooth bounded open subset of $\mathbb{R}^{m}$, then our theorem reduces essentially to a well-known classical result (e.g., 27]).

More recently, G. Grubb 22 has established a general $L_{p}$ theory for parabolic pseudo-differential boundary value problems acting on sections of vector bundles (also see Section IV.4.1 in [23]). It applies to a class of noncompact manifolds, called 'admissible' and being introduced in G. Grubb and N.J. Kokholm 24. It is a subclass of the above family of manifolds with funnel-like ends, namely a family of manifolds with conical ends. Of course, aside from the requirements on the manifold, differential boundary value problems of the form considered in the present paper constitute a very particular subcase of Grubb's general class. However, in order to apply the results of 22 to 3.1 we have to require that $(\mathcal{A}, \mathcal{B})$ has $C^{\infty}$ coefficients. In contrast, we impose in essence minimal regularity assumptions on $(\mathcal{A}, \mathcal{B})$. This is important for the study of quasilinear equations on the basis of the linear theorems proved here.

Now we suppose that $M$ is a noncompact uniformly regular Riemannian manifold not belonging to the class of manifolds with funnel-like ends. This is the case, in particular, if $M$ has no boundary, is complete, and has bounded geometry. There is a tremendous amount of literature on heat equations for such manifolds, most of which is an $L_{2}$ theory and is concerned with kernel estimates and spectral theory (see, for example, E.B. Davies [16] or A. Grigor'yan [21] and the references therein). There are a few papers dealing with (semilinear) parabolic equations on noncompact complete Riemannian manifolds under various curvature assumptions which are based on heat kernel estimates (e.g., Qi S. Zhang [35], 36], A.L. Mazzucato and V. Nistor [29], F. Punzo 31, 32, C. Bandle, F. Punzo, and A. Tesei 14). In all these papers either the top-order part is the Laplace-Beltrami operator or smooth leading order coefficients are required.

Except for a recent paper by Y. Shao and G. Simonett 33, the author is not aware of any result on parabolic equations on noncompact manifolds 
which do not rely on heat kernel techniques, leave alone noncompact manifolds with noncompact boundary. In [33 the authors, building on [5] and 6], establish a Hölder space existence theorem for autonomous nonlinear parabolic equations on uniformly regular manifolds without boundary. As an application they show that the solutions of the Yamabe flow instantaneously regularize and become real analytic in space and time.

A prototypical example to which our results apply is furnished by an $m$-dimensional Riemannian submanifold $M_{\mathrm{H}}=\left(M_{\mathrm{H}}, g_{\mathrm{H}}\right)$ of the hyperbolic space $\mathrm{H}^{m}$ represented by the Poincaré model. More specifically, we denote by $\mathbb{B}^{m}$ the open unit ball in $\mathbb{R}^{m}$ with closure $\overline{\mathbb{B}}^{m}$ and boundary $S^{m-1}$, the $(m-1)$-sphere. Then $\mathbf{H}=\mathbf{H}^{m}=\left(\mathbb{B}^{m}, g_{\mathbf{H}}\right)$, where $g_{\mathbf{H}}=4 g_{m} /\left(1-|x|^{2}\right)^{2}$ for $x \in \mathbb{B}^{m}$. If $\partial M_{\mathrm{H}}$ is not compact, then we assume that its closure in $\overline{\mathbb{B}}^{m}$ intersects $S^{m-1}$ transversally and that this intersection is the boundary of an $(m-1)$-dimensional Riemannian submanifold of $S^{m-1}$. Informally expressed this means, in particular, that $M_{\mathrm{H}}$ 'does not collapse at infinity'.

Writing $\operatorname{div}_{\mathrm{H}}$ for $\operatorname{div}_{g_{\mathrm{H}}}$, etc., problem (3.1) is on $M_{\mathrm{H}} \times J$ given by

$$
\mathcal{A}_{\mathrm{H}} u:=-\operatorname{div}_{\mathrm{H}}\left(a \cdot \operatorname{grad}_{\mathrm{H}} u\right)+\left(\vec{a} \mid \operatorname{grad}_{\mathrm{H}} u\right)_{\mathrm{H}}+a_{0} u
$$

and

$$
\mathcal{B}_{\mathrm{H}} u:= \begin{cases}\gamma u & \text { on } \partial_{0} M_{\mathrm{H}}, \\ \left(\nu_{\partial M_{\mathrm{H}}} \mid \gamma\left(a \cdot \operatorname{grad}_{\mathrm{H}} u\right)\right)_{\mathrm{H}} & \text { on } \partial_{1} M_{\mathrm{H}} .\end{cases}
$$

Using the fact that $g_{\mathrm{H}}$ is conformal to $g_{m}$ we can express $\mathcal{A}_{\mathrm{H}}$ and $\mathcal{B}_{\mathrm{H}}$ in terms of $g_{m}$, that is, as differential operators on $M:=\left(M_{\mathrm{H}}, g_{m}\right)$. In fact, writing $\operatorname{div}=\operatorname{div}_{g_{m}}$, etc., we find with $\rho(x):=\left(1-|x|^{2}\right) / 2$

$$
\begin{aligned}
\operatorname{div}_{\mathrm{H}}\left(a \cdot \operatorname{grad}_{\mathrm{H}} u\right) & =\rho^{m} \partial_{i}\left(\rho^{2-m} a_{j}^{i} \delta^{j k} \partial_{k} u\right)=\rho^{m} \operatorname{div}\left(\rho^{2-m} a \cdot \operatorname{grad} u\right) \\
& =\operatorname{div}\left(\rho^{2} a \cdot \operatorname{grad} u\right)-m(\rho a \cdot \operatorname{grad} \rho \mid \operatorname{grad} u)
\end{aligned}
$$

and $\left(\vec{a} \mid \operatorname{grad}_{\mathrm{H}} u\right)_{\mathrm{H}}=(\vec{a} \mid \operatorname{grad} u)$. Moreover, $\nu_{\partial M_{\mathrm{H}}}=\rho \nu$ and, consequently,

$$
\left(\nu_{\mathrm{H}} \mid \gamma\left(a \cdot \operatorname{grad}_{\mathrm{H}} u\right)\right)_{\mathrm{H}}=\rho(\nu \mid \gamma(a \cdot \operatorname{grad} u)) .
$$

This shows that the initial boundary value problem

$$
\partial_{t} u+\mathcal{A}_{\mathrm{H}} u=f \text { on } M_{\mathrm{H}} \times J, \quad \mathcal{B}_{\mathrm{H}} u=h \text { on } \partial M_{\mathrm{H}} \times J, \quad u=u_{0} \text { on } M_{\mathrm{H}} \times\{0\}
$$

can be seen as a degenerate initial boundary value problem on the 'underlying' Euclidean manifold $M$. Note that $M$ is not a uniformly regular Riemannian manifold, even if $\partial M=\emptyset$, that is, $M=\mathbb{B}^{m}$, since it cannot be covered by an atlas $\mathfrak{K}$ whose coordinate patches are uniformly comparable in size and such that a uniform shrinking of $\mathfrak{K}$ is still an atlas.

\section{Singular Riemannian Manifolds and Weighted Function Spaces}

Generalizing the preceding example we are led to the concept of singular Riemannian manifolds. Informally speaking, such a manifold is characterized by a singularity function $\rho \in C^{\infty}(M,(0, \infty))$ such that the conformal metric 
$\hat{g}:=g / \rho^{2}$ gives rise to a uniformly regular Riemannian manifold $\hat{M}:=(M, \hat{g})$. To be precise:

Let $M$ be an $m$-dimensional uniformly regular manifold. A pair $(\rho, \mathfrak{K})$ is a singularity datum for $M$ if $\rho \in C^{\infty}(M,(0, \infty))$ and $\mathfrak{K}$ is a uniformly regular atlas such that

$$
\begin{aligned}
& \left\|\kappa_{*} \rho\right\|_{k, \infty} \leq c(k) \rho_{\kappa}, \quad \kappa \in \mathfrak{K}, \quad k \in \mathbb{N}, \\
& \text { where } \rho_{\kappa}:=\kappa_{*} \rho(0)=\rho\left(\kappa^{-1}(0)\right) ; \\
& \rho \mid U_{\kappa} \sim \rho_{\kappa}, \quad \kappa \in \mathfrak{K} .
\end{aligned}
$$

Two singularity data $(\rho, \mathfrak{K})$ and $(\tilde{\rho}, \tilde{\mathfrak{K}})$ are equivalent, $(\rho, \mathfrak{K}) \approx(\tilde{\rho}, \tilde{\mathfrak{K}})$, if $\rho \sim \tilde{\rho}$ and $\mathfrak{K} \approx \tilde{\mathfrak{K}}$.

A singularity structure, $\mathfrak{S}(M)$, for $M$ is a maximal family of equivalent singularity data. A singularity function for $M$ is a $\rho \in C^{\infty}(M,(0, \infty))$ such that there exists an atlas $\mathfrak{K}$ with $(\rho, \mathfrak{K}) \in \mathfrak{S}(M)$. The set of all singularity functions is the singularity type of $M$. It is convenient to denote it by $\llbracket \rho \rrbracket$, where $\rho$ is one of its representatives.

A singular Riemannian manifold of type $\llbracket \rho \rrbracket$ is a Riemannian manifold $(M, g)$ such that

(i) $\quad M$ is uniformly regular and endowed with a singularity structure $\mathfrak{S}(M)$ of singularity type $\llbracket \rho \rrbracket$;

(ii) $\quad\left(M, g / \rho^{2}\right)$ is a uniformly regular Riemannian manifold.

This definition is independent of the particular choice of $\rho$ in the following sense: Let $(\tilde{\rho}, \tilde{\mathfrak{K}}) \approx(\rho, \mathfrak{K})$. Then it follows from (2.5) (ii), (iii) and (4.1) that $\left(M, g / \tilde{\rho}^{2}\right)$ is a uniformly regular Riemannian manifold and $g / \tilde{\rho}^{2} \sim g / \rho^{2}$. In 7$]$ it is shown that (4.1) (i) is equivalent to

$$
d \log \rho \in B C^{\infty}\left(T^{*} \hat{M}\right) .
$$

In [9] there is carried out a detailed study of singular Riemannian manifolds. We refer the reader to that paper for proofs of the following examples.

Examples 4.1. (a) A uniformly regular Riemannian manifold is singular of type $\llbracket \mathbf{1} \rrbracket$, and conversely.

(b) Let $\Gamma$ be a union of connected components of $\partial M$ and $m \geq 2$. We endow $\Gamma$ with the induced Riemannian metric $\dot{g}:=i^{*} g$, where $i: \Gamma \hookrightarrow M$ is the natural embedding. Let $\mathfrak{K}$ be a uniformly regular atlas for $M$. For $\kappa \in \mathfrak{K}_{\Gamma}$ we set $U_{\dot{\kappa}}:=\partial U_{\kappa}:=U_{\kappa} \cap \partial M=U_{\kappa} \cap \Gamma$ and $\dot{\kappa}:=\iota_{0} \circ\left(i^{*} \kappa\right): U_{\dot{\kappa}} \rightarrow \mathbb{R}^{m-1}$ with $\iota_{0}:\{0\} \times \mathbb{R}^{m-1} \rightarrow \mathbb{R}^{m-1}, \quad\left(0, x^{\prime}\right) \mapsto x^{\prime}$. Then $\dot{\mathfrak{K}}:=\left\{\dot{\kappa} ; \kappa \in \mathfrak{K}_{\Gamma}\right\}$ is a uniformly regular atlas for $\Gamma$, the one induced by $\mathfrak{K}$.

Suppose $(M, g)$ is a singular Riemannian manifold of type $\llbracket \rho \rrbracket$. We set $\dot{\rho}:=i^{*} \rho=\rho \mid \Gamma$. Then $(\dot{\rho}, \dot{\mathfrak{K}})$ is a singularity datum for $\Gamma$. Thus it defines a singular structure $\dot{\mathfrak{S}}(\Gamma)$ for $\Gamma$, the one induced by $\mathfrak{S}(M)$. Furthermore, $\left(\Gamma, \dot{g} / \dot{\rho}^{2}\right)$ is a singular Riemannian manifold of type $\llbracket \dot{\rho} \rrbracket$ (and dimension $m-1$ ). It is always understood that $\Gamma$ is endowed with the singular structure induced by the one of $M$. 
(c) Let $(\tilde{M}, \tilde{g})$ be a Riemannian manifold and $f: M \rightarrow \tilde{M}$ an isometric diffeomorphism, that is, $\tilde{g}=f_{*} g$. Suppose $(M, g)$ is singular of type $\llbracket \rho \rrbracket$ with singularity datum $(\rho, \mathfrak{K})$. Set $f_{*} \mathfrak{K}:=\left\{f_{*} \kappa ; \kappa \in \mathfrak{K}\right\}$. Then the pair $\left(f_{*} \rho, f_{*} \mathfrak{K}\right)$ is a singularity datum for $(\tilde{M}, \tilde{g})$ and the latter is a singular Riemannian manifold of type $\llbracket f_{*} \rho \rrbracket$.

(d) Suppose $S \subset U \subset M$, where $S$ is closed and $U$ is open in $M$. Assume $\rho \in C^{\infty}(U,(0, \infty))$ and $\mathfrak{K}$ is a uniformly regular atlas for $U$ on $S$ such that (4.1) holds for $\mathfrak{K}_{S}$. Then $(\rho, \mathfrak{K})$ is a singularity structure for $U$ on $S$. Two such singularity structures $(\rho, \mathfrak{K})$ and $(\tilde{\rho}, \tilde{\mathfrak{K}})$ are equivalent on $S$ if $\rho \sim \tilde{\rho}$ and $\mathfrak{K}$ and $\tilde{\mathfrak{K}}$ are equivalent on $S$. This defines a singularity structure for $U$ on $S$ of type $\llbracket \rho \rrbracket$. Then $U$ is a singular Riemannian manifold on $S$ of type $\llbracket \rho \rrbracket$ if it is endowed with a singularity structure on $S$ of type $\llbracket \rho \rrbracket$ and $\left(U, g / \rho^{2}\right)$ is a uniformly regular Riemannian manifold on $S$.

Assume $S_{j} \subset U_{j} \subset M$ and $U_{j}$ is a uniformly regular Riemannian manifold on $S_{j}$ of type $\llbracket \rho_{j} \rrbracket$ for $0 \leq j \leq \ell$. Let $\left(\rho_{j}, \mathfrak{K}_{j}\right)$ be a singularity structure for $U_{j}$ on $S_{j}$ and assume that $(\alpha)$ and ( $\beta$ ) of Example 3.5(f) apply and

$$
\rho_{i}\left|\left(S_{i} \cap S_{j}\right) \sim \rho_{j}\right|\left(S_{i} \cap S_{j}\right), \quad 0 \leq i<j \leq \ell .
$$

Then there exists $\rho \in C^{\infty}(M,(0, \infty))$ such that $\rho\left|S_{j} \sim \rho_{j}\right| S_{j}$ for $0 \leq j \leq \ell$ and $(\rho, \mathfrak{K})$ is a singularity datum for $M$, where $\mathfrak{K}=\mathfrak{K}_{0} \cup \cdots \cup \mathfrak{K}_{\ell}$. Furthermore, $M$ is a singular Riemannian manifold of type $\llbracket \rho \rrbracket$. It is said to be obtained by patching together the singular Riemannian manifolds $U_{j}$ on $S_{j}$ of type $\llbracket \rho_{j} \rrbracket$.

(e) Let $d \geq 2$ and suppose $B$ is a $b$-dimensional compact Riemannian submanifold of $\mathbb{R}^{d-1}$. For a nonempty subinterval $I$ of $(0,1)$ and $\alpha \geq 1$ we set

$$
C_{\alpha}^{d}(I, B):=\left\{\left(t, t^{\alpha} y\right) ; t \in I, y \in B\right\} \subset \mathbb{R} \times \mathbb{R}^{d-1}=\mathbb{R}^{d} .
$$

We endow $C_{\alpha}^{d}(B):=C_{\alpha}^{d}((0,1), B)$ with the metric induced by $\mathbb{R}^{d}$. It is called model $\alpha$-cusp over (the base) $B$ in $\mathbb{R}^{d}$. Note that a 1-cusp is a cone.

For $\ell \in \mathbb{N}$ we set $C_{\alpha, \ell}^{d}(I, B):=C_{\alpha}^{d}(I, B)$ if $\ell=0$, and

$$
C_{\alpha, \ell}^{d}(I, B):=C_{\alpha}^{d}(I, B) \times I Q^{\ell}, \quad \ell>0,
$$

where $I Q^{\ell}=\left\{t z \in \mathbb{R}^{\ell} ; t \in I, z \in Q^{\ell}\right\}$. Then $C_{\alpha, \ell}^{d}(B):=C_{\alpha, \ell}^{d}((0,1), B)$ is a $(1+b+\ell)$-dimensional Riemannian submanifold of $\mathbb{R}^{d} \times \mathbb{R}^{\ell}=\mathbb{R}^{d+\ell}$, a model $(\alpha, \ell)$-wedge over $B$, also called model $\ell$-wedge over $C_{\alpha}^{d}(B)$. Thus every model cusp is a model wedge, a 0 -wedge. Every $(\alpha, \ell)$-wedge $C=C_{\alpha, \ell}^{d}(B)$ is a singular Riemannian manifold on $S:=C_{\alpha, \ell}^{d}((0,3 / 4], B)$ of type $\llbracket R_{\alpha} \rrbracket$, where the cusp characteristic $R_{\alpha}$ is defined by $R_{\alpha}(x):=t^{\alpha}$ for $x=(t, y, z) \in C$ with $y \in Q^{\ell}$.

(f) Let $U$ be open in $M$ and set $C:=C_{\alpha, \ell}^{d}(B)$ and $S:=C_{\alpha, \ell}^{d}((0,3 / 4], B)$ with $\ell:=m-1-b \geq 0$. Suppose that $\varphi: U \rightarrow C$ is a diffeomorphism such that $\left(\varphi_{*} g\right)\left|S \sim g_{C}\right| S$. Then $U$ is a singular Riemannian manifold on $\varphi^{-1}(S)$ of type $\llbracket \varphi^{*} R_{\alpha} \rrbracket$. It is said to be an $(\alpha, \ell)$-wedge represented by $\varphi$. 
(g) Let $d \geq m$ and let $B$ be an $(m-1)$-dimensional compact submanifold of $\mathbb{R}^{d-1}$. For a nonempty subinterval $I$ of $(1, \infty)$ and $\alpha<0$ we set

$$
K_{\alpha}^{d}(I, B):=\left\{\left(t, t^{\alpha} y\right) ; t \in I, y \in B\right\} \subset \mathbb{R} \times \mathbb{R}^{d-1}=\mathbb{R}^{d} .
$$

Then $K_{\alpha}^{d}(B):=K_{\alpha}^{d}((1, \infty), B)$ is considered as an $m$-dimensional Riemannian submanifold of $\mathbb{R}^{d}$, an infinite $\alpha$-cusp over $B$ in $\mathbb{R}^{d}$. Its cusp characteristic $R_{\alpha}$ is given by $R_{\alpha}(x):=t^{\alpha}$ for $x=\left(t, t^{\alpha} y\right) \in K_{\alpha}^{d}(B)$. It holds that $K_{\alpha}^{d}(B)$ is a singular Riemannian manifold on $K_{\alpha}^{d}([2, \infty), B)$ of type $\llbracket R_{\alpha} \rrbracket$.

(h) Let $U$ be open in $M$ and let $K:=K_{\alpha}^{d}(B)$ be an infinite $\alpha$-cusp over $B$ in $\mathbb{R}^{d}$. Set $S:=K_{\alpha}^{d}([2, \infty), B)$. Let $\varphi: U \rightarrow K$ be a diffeomorphism satisfying

$(\alpha) \overline{U \backslash \varphi^{-1}(S)} \cap S=\varphi^{-1}\left(K_{\alpha}^{d}(\{2\}, B)\right) ;$

$(\beta)\left(\varphi_{*} g\right)\left|S \sim g_{K}\right| S$.

Then $U$ is a singular Riemannian manifold on $\varphi^{-1}(S)$ of type $\llbracket \varphi^{*} R_{\alpha} \rrbracket$. Furthermore, $M$ is said to have in $U$ an infinite $\alpha$-cusp (more precisely: $(\alpha, B)$ cusp) represented by $\varphi$.

(i) Assume that $M$ is an $m$-dimensional Riemannian submanifold for $\mathbb{R}^{n}$ for some $n \geq m$. Then $\mathcal{S}(M):=\bar{M} \backslash M$, where $\bar{M}$ is the closure of $M$ in $\mathbb{R}^{n}$, is the singularity set of $M$. It is independent of $n$ since the closure of $M$ in $\mathbb{R}^{\tilde{n}}$ with $\tilde{n}>n$ and $\mathbb{R}^{n}=\mathbb{R}^{n} \times\{0\} \subset \mathbb{R}^{\tilde{n}}$ equals $\bar{M}$ also.

Suppose $\Sigma$ is a connected component of $\mathcal{S}(M)$ with the following properties:

$(\alpha)$ it is an $\ell$-dimensional compact Riemannian submanifold of $\mathbb{R}^{n}$ without boundary, where $\ell \in\{0, \ldots, m-1\}$;

$(\beta)$ there exist $\alpha \geq 1$, a compact $(m-\ell-1)$-dimensional Riemannian submanifold $B$ of $\mathbb{R}^{d}$ with $d \geq m-\ell$, and for each $p \in \Sigma$ a normalized chart $\Phi_{p}$ for $\mathbb{R}^{n}$ at $p$ such that, setting $V_{p}:=\operatorname{dom}\left(\Phi_{h}\right)$,

$$
\Phi_{p}\left(M \cap V_{p}\right)=C_{(\alpha, \ell)}^{d}(B) \times\{0\} \subset \mathbb{R}^{d+\ell} \times \mathbb{R}^{n-d-\ell}=\mathbb{R}^{n}
$$

and

$$
\Phi_{p}\left(\Sigma \cap V_{p}\right)=\left(\{0\} \times Q^{\ell}\right) \times\{0\} ;
$$

( $\gamma) U_{p}:=M \cap V_{p}$ is an $(\alpha, \ell)$-wedge represented by $\varphi_{p}:=\Phi_{p} \mid U_{p}$.

Then $M$ is said to possess a smooth cuspidal singularity of type $(\alpha, \ell)$ (more precisely: $(\alpha, \ell, B))$ near $\Sigma$.

Let $\Sigma \subset \mathcal{S}(M)$ and assume $M$ has a smooth cuspidal singularity of type $(\alpha, \ell)$ near $\Sigma$. Also assume that there exist relatively compact open neighborhoods $V$ and $W$ of $\Sigma$ in $\mathbb{R}^{n}$ with $\bar{W} \subset V$ possessing the following properties: set $U:=V \cap M$ and $S:=\bar{W} \cap M$. Then there is $\rho_{\alpha} \in C^{\infty}(U,(0, \infty))$ such that

$$
\rho_{\alpha}\left|\left(S \cap U_{p}\right) \sim \varphi_{p}^{*} R_{\alpha}\right|\left(S \cap U_{p}\right), \quad p \in \Sigma,
$$

and $U$ is on $S$ a singular Riemannian manifold of type $\llbracket \rho_{\alpha} \rrbracket$. Loosely speaking: $\Sigma$ is then said to be a smooth $(\alpha, \ell)$-wedge, more precisely, a smooth $(\alpha, \ell, B)$ wedge. It is a smooth $\alpha$-cusp, respectively $(\alpha, B)$-cusp, if $\ell=0$. Note that near every smooth $\left(\alpha, S^{m-1}\right)$-cusp $M$ looks locally like $\mathbb{R}^{m} \backslash\{0\}$ near 0 . (In this case we choose $d=m+1$.) 
(j) Let $M$ be an $m$-dimensional Riemannian submanifold of $\mathbb{R}^{n}$ for some $n \geq m$. Suppose:

$(\alpha) \mathcal{S}(M)$ is compact and for each connected component $\Sigma$ of $\mathcal{S}(M)$ there exist $\alpha_{\Sigma} \geq 1, \quad \ell_{\Sigma} \in\{0, \ldots, m-1\}$, and a compact $(m-\ell-1)$ dimensional submanifold $B_{\Sigma}$ of $\mathbb{R}^{d}$, where $d \geq m-\ell$, such that $M$ has a smooth cuspidal singularity of type $\left(\alpha_{\Sigma}, \ell_{\Sigma}, B_{\Sigma}\right)$ near $\Sigma$;

$(\beta)$ there are $k \in \mathbb{N}$ and for each $i \in\{1, \ldots, k\}$ an open subset $U_{i}$ of $M$, $\alpha_{i} \in(-\infty, 0)$, an $(m-1)$-dimensional compact Riemannian submanifold $B_{i}$ of $\mathbb{R}^{d}$ for some $d \geq m$, and a diffeomorphism $\varphi_{i}: U_{j} \rightarrow K_{\alpha_{i}}^{d}\left(B_{j}\right)$ such that $M$ has in $U_{i}$ an infinite $\left(\alpha_{i}, B_{i}\right)$-cusp represented by $\varphi_{i}$;

$(\gamma) U_{i} \cap U_{j}=\emptyset$ for $1 \leq i<j \leq k, M \backslash\left(U_{1} \cup \cdots \cup U_{k}\right)$ is relatively compact in $\mathbb{R}^{n}$, and $\mathcal{S}(M) \backslash\left(U_{1} \cup \cdots \cup U_{k}\right)=\mathcal{S}(M)$.

Then $M$ is said to be a manifold with cuspidal singularities. Note that $M$ is relatively compact in $\mathbb{R}^{n}$ if $k=0$.

Every manifold with cuspidal singularities is a singular Riemannian manifold of type $\llbracket \rho \rrbracket$, where $\rho \sim \rho_{\alpha}$ near a smooth $(\alpha, \ell)$-wedge $\Sigma \subset \mathcal{S}(M)$, $\rho \sim \varphi_{i}^{*} R_{\alpha_{i}}$ on $U_{i}, \quad 1 \leq i \leq k$, and $\rho \sim 1$ away from the singularities.

The qualifier 'smooth' in the preceding definitions refers to the fact that the bases of the cusps are uniformly regular. If they are singular Riemannian manifolds themselves then we get manifolds with cuspidal corners of various orders. For this we refer to [9] as well.

B. Ammann, R. Lauter, and V. Nistor [1] introduce a class of noncompact Riemannian manifolds, termed Lie manifolds, in order to establish regularity properties of solutions to elliptic boundary value problems on polyhedral domains; also see B. Ammann, A.D. Ionescu and V. Nistor [10, B. Ammann and V. Nistor [12], and the references therein, as well as the survey by C. Bacuta, A.L. Mazzucato, and V. Nistor [13. These authors use a desingularization technique by which they introduce conformal metrics $g / \rho^{2}$, where $\rho$ is the distance to the singular set.

Let $M=(M, g)$ be a singular Riemannian manifold of type $\llbracket \rho \rrbracket$. Then we can apply the results of Sections 2 and 3 to $\hat{M}$, where we have to use $\hat{\nabla}:=\nabla_{\hat{g}}$, of course. Fortunately, since $\hat{g}$ is conformal to $g$ we can express all spaces and operators in terms of $g$, so that $\hat{M}$ does not appear in the final results.

Specifically, set $V:=V_{\tau}^{\sigma}$ and let $\lambda \in \mathbb{R}$. Then the weighted Sobolev space $W_{p}^{k, \lambda}(V ; \rho)$ is for $k \in \mathbb{N}$ the completion of $\mathcal{D}(V)$ in $L_{1, \text { loc }}(V)$ with respect to the norm

$$
u \mapsto\left(\sum_{j=0}^{k}\left\|\rho^{\lambda+j+\tau-\sigma}\left|\nabla^{j} u\right|_{g_{\sigma}^{\tau+j}}\right\|_{L_{p}(V)}^{p}\right)^{1 / p} .
$$

Weighted Slobodeckii spaces $W_{p}^{s, \lambda}(V ; \rho)$ are for $k<s<k+1$ again defined by interpolation, that is, by replacing $W_{p}^{\ell}(V)$ in 2.1) by $W_{p}^{\ell, \lambda}(V ; \rho)$ for $\ell \in\{k, k+1\}$. 
Analogously, $B^{\lambda}(V ; \rho)$ is the vector space of all sections $u$ of $V$ such that $\rho^{\lambda+\tau-\sigma}|u|_{g_{\sigma}^{\tau}} \in B(M)$. The norm $u \mapsto\left\|\rho^{\lambda+\tau-\sigma}|u|_{g_{\sigma}^{\tau}}\right\|_{\infty}$ makes it a Banach space. If $k \in \mathbb{N}$, then $B^{k, \lambda}(V ; \rho)$ is the Banach space of all $u \in C^{k}(V)$ for which

$$
\max _{0 \leq j \leq k}\left\|\rho^{\lambda+j+\tau-\sigma}\left|\nabla^{j} u\right|_{g_{\sigma}^{\tau+j}}\right\|_{\infty}
$$

is finite, endowed with this norm. Furthermore, $b c^{k, \lambda}(V ; \rho)$ is the closure of $B C^{\infty, \lambda}(V ; \rho):=\bigcap_{k} B C^{k}(V ; \rho)$ in $B C^{k, \lambda}(V ; \rho)$. Then weighted Besov-Hölder spaces $B_{\infty}^{s, \lambda}(V ; \rho)$ are defined by interpolation in complete analogy to (2.2).

Weighted $L_{2}$ Sobolev spaces of this type have been introduced by V.A. Kondrat'ev 25] in the study of elliptic boundary value problems on domains with singular points. Since then they have been used by numerous authors, predominantly in an Euclidean $L_{2}$ setting. A detailed study of the $L_{p}$ case and references are found in [6].

We set

$$
\begin{gathered}
W_{p}^{s}(V ; \rho):=W_{p}^{s, 0}(V ; \rho), \quad B C^{k}(V ; \rho):=B C^{k, 0}(V ; \rho), \\
B_{\infty}^{s}(V ; \rho):=B_{\infty}^{s, 0}(V ; \rho) .
\end{gathered}
$$

In [7] it is proved that

$$
\begin{gathered}
W_{p}^{s}(\hat{V}) \doteq W_{p}^{s,-m / p}(V ; \rho), \quad B C^{k}(\hat{V}) \doteq B C^{k}(V ; \rho), \\
B_{\infty}^{s}(\hat{V}) \doteq B_{\infty}^{s}(V ; \rho),
\end{gathered}
$$

where $\doteq$ means 'equal except for equivalent norms' and $\hat{V}:=T_{\tau}^{\sigma} \hat{M}$. In [7] it is also shown that $\left(u \mapsto \rho^{\lambda} u\right)$ belongs to

$$
\mathcal{L} \operatorname{is}\left(W_{p}^{s, \lambda^{\prime}+\lambda}(V ; \rho), W_{p}^{s, \lambda^{\prime}}(V ; \rho)\right) \cap \mathcal{L} \operatorname{is}\left(B_{\infty}^{s, \lambda^{\prime}+\lambda}(V ; \rho), B_{\infty}^{s, \lambda^{\prime}}(V ; \rho)\right)
$$

for $\lambda, \lambda^{\prime} \in \mathbb{R}$, and $\left(u \mapsto \rho^{\lambda} u\right)^{-1}=\left(v \mapsto \rho^{-\lambda} v\right)$. Thus it suffices to study the spaces $W_{p}^{s}(\hat{V})$ and $B_{\infty}^{s}(\hat{V})$ since by this isomorphism and by (4.4) we can transfer all properties from $W_{p}^{s}(\hat{V})$ onto $W_{p}^{s, \lambda}(V ; \rho)$ and from $B_{\infty}^{s}(\hat{V})$ onto $B_{\infty}^{s, \lambda}(V ; \rho)$. Alternatively, we can refer directly to [6].

Anisotropic weighted Sobolev-Slobodeckii spaces are defined for $s \geq 0$ by

$$
W_{p}^{(s, s / 2), \lambda}(V \times J ; \rho):=L_{p}\left(J, W_{p}^{s, \lambda}(V ; \rho)\right) \cap W_{p}^{s / 2}\left(J, L_{p}^{\lambda}(V ; \rho)\right) .
$$

Analogously, we introduce anisotropic Besov-Hölder spaces for $s>0$ by

$$
\left.B_{\infty}^{(s, s / 2), \lambda}(V ; \rho)\right):=B\left(J, B_{\infty}^{s, \lambda}(V ; \rho)\right) \cap B_{\infty}^{s / 2}\left(J, B^{\lambda}(V ; \rho)\right) .
$$

Again, we omit the superscript $\lambda$ if it equals zero. It is obvious from the above that all embedding, interpolation, and trace theorems, etc. proved in 5 ] carry over to the present setting using natural adaptions. It is also clear that (4.4) implies

$$
W_{p}^{(s, s / 2)}(\hat{V}) \doteq W_{p}^{(s, s / 2),-m / p}(V ; \rho) .
$$


Furthermore,

$$
\left(u \mapsto \rho^{\lambda} u\right) \in \mathcal{L} \operatorname{is}\left(W_{p}^{(s, s / 2), \lambda^{\prime}+\lambda}(V ; \rho), W_{p}^{(s, s / 2), \lambda^{\prime}}(V ; \rho)\right)
$$

for $\lambda, \lambda^{\prime} \in \mathbb{R}$ is a consequence of (4.5).

\section{Degenerate Parabolic Problems}

In this section we study problem 3.1) in the case of singular Riemannian manifolds. It turns out that in this situation Theorem 3.1 leads to an isomorphism theorem for degenerate parabolic initial boundary value problems on weighted Sobolev spaces.

Let $M=(M, g)$ be a singular Riemannian manifold of type $\llbracket \rho \rrbracket$ and $\lambda \in \mathbb{R}$. Similarly as in Section 3. we introduce data spaces which are now weighted and $\lambda$-dependent. To simplify the presentation we restrict ourselves to the setting of strong $L_{p}$ solutions. Thus we put

$$
\begin{aligned}
& W_{p}^{(2-\vec{\delta}-1 / p)(1,1 / 2), \lambda+\vec{\delta}+1 / p}\left(\partial M_{T} ; \dot{\rho}\right) \\
& \quad:=W_{p}^{(2-1 / p)(1,1 / 2), \lambda+1 / p}\left(\partial_{0} M_{T} ; \dot{\rho}\right) \times W_{p}^{(1-1 / p)(1,1 / 2), \lambda+1+1 / p}\left(\partial_{1} M_{T} ; \dot{\rho}\right)
\end{aligned}
$$

and

$$
\begin{aligned}
& \mathcal{W}_{p}^{(2,1), \lambda}\left(M_{T} ; \rho\right) \\
& :=L_{p}^{\lambda}\left(M_{T} ; \rho\right) \times W_{p}^{(2-\vec{\delta}-1 / p)(1,1 / 2), \lambda+\vec{\delta}+1 / p}\left(\partial M_{T} ; \dot{\rho}\right) \times W_{p}^{2-2 / p, \lambda}(M ; \rho) .
\end{aligned}
$$

Similarly as before, $\mathcal{W}_{p, \mathrm{cc}}^{(2,1), \lambda}\left(M_{T} ; \rho\right)$ is the linear subspace hereof consisting of all $\left(f, h, u_{0}\right)$ satisfying the compatibility conditions (3.4).

The differential operator (3.2) is uniformly strongly $\rho$-elliptic if $a(\cdot, t)$ is symmetric for $t \in J$ and there exists a constant $\varepsilon>0$ such that

$$
(a(q, t) \cdot X \mid X)_{g(q)} \geq \varepsilon \rho^{2}(q)|X|_{g(q)}^{2}, \quad X \in T_{q} M, \quad q \in M, \quad t \in J .
$$

Henceforth, we say that $(\mathcal{A}, \mathcal{B})$ is a $\rho$-regular uniformly $\rho$-elliptic boundary value problem on $M_{T}$ if $\mathcal{A}$ is uniformly strongly $\rho$-elliptic,

$$
\begin{gathered}
a \in B C^{(1,1 / 2),-2}\left(T_{1}^{1} M \times J ; \rho\right), \quad \vec{a} \in L_{\infty}(T M \times J ; \rho), \\
a_{0} \in L_{\infty}\left(M_{T}\right),
\end{gathered}
$$

and

$$
b_{0} \in B C^{(1,1 / 2),-1}\left(\partial_{1} M_{T}\right) .
$$

If $\rho=\mathbf{1}$, then $(\mathcal{A}, \mathcal{B})$ is simply called regularly uniformly elliptic. Note that the first part of (5.2) implies $|a|_{g_{1}^{1}} \leq c \rho^{2}$. Using this and the symmetry of $a(\cdot, t)$ we see that (5.1) is equivalent to the existence of $\varepsilon \in(0,1)$ with

$$
\varepsilon \rho^{2}(q)|X|_{g(q)}^{2} \leq(a(q, t) \cdot X \mid X)_{g(q)} \leq \rho^{2}(q)|X|_{g(q)}^{2} / \varepsilon
$$

for $X \in T_{q} M, q \in M$, and $t \in J$.

Now we can formulate the following isomorphism theorem for degenerate parabolic equations. 
Theorem 5.1. Let $M$ be a singular Riemannian manifold of type $\rho \rrbracket$ and $p \notin\{3 / 2,3\}$. Suppose that $(\mathcal{A}, \mathcal{B})$ is a $\rho$-regular uniformly $\rho$-elliptic boundary value problem on $M_{T}$ and $\lambda \in \mathbb{R}$.

Then $\mathcal{W}_{p, \mathrm{cc}}^{(2,1), \lambda}\left(M_{T} ; \rho\right)$ is closed and

$$
\left(\partial+\mathcal{A}, \mathcal{B}, \gamma_{0}\right) \in \mathcal{L} \operatorname{is}\left(W_{p}^{(2,1), \lambda}\left(M_{T} ; \rho\right), \mathcal{W}_{p, \mathrm{cc}}^{(2,1), \lambda}\left(M_{T} ; \rho\right)\right)
$$

The proof of this theorem is given later in this section. First we derive an analogue of Theorem 3.4. For this we define

$$
W_{p, \mathcal{B}}^{s, \lambda}(M ; \rho), \quad s \in[0,2] \backslash\{1 / p, 1+1 / p\},
$$

by replacing $W_{p}^{s}(M)$ in (1.4) by $W_{p}^{s, \lambda}(M ; \rho)$.

Theorem 5.2. Let $M$ be a singular Riemannian manifold of type $\rho \rrbracket$ and $p \notin\{3 / 2,3\}$. Suppose $(\mathcal{A}, \mathcal{B})$ is an autonomous $\rho$-regular uniformly $\rho$-elliptic boundary value problem on $M_{T}$ and $\lambda \in \mathbb{R}$. Set $A^{\lambda}:=\mathcal{A} \mid W_{p, \mathcal{B}}^{2, \lambda}(M ; \rho)$, considered as an unbounded linear operator in $L_{p}^{\lambda}(M ; \rho)$. Then $-A^{\lambda}$ generates a strongly continuous analytic semigroup on $L_{p}^{\lambda}(M ; \rho)$ and has the property of maximal regularity, that is, $(\partial+\mathcal{A}, \gamma)$ belongs to

$$
\mathcal{L} \operatorname{is}\left(L_{p}\left(J, W_{p, \mathcal{B}}^{2, \lambda}(M ; \rho)\right) \cap W_{p}^{1}\left(J, L_{p}^{\lambda}(M ; \rho)\right), L_{p}^{\lambda}(M ; \rho) \times W_{p, \mathcal{B}}^{2-2 / p, \lambda}(M)\right) .
$$

Proof. This follows from Theorem 5.1 by the arguments which led from Theorem 3.1 to Theorem 3.4 .

Corollary 5.3. Set $A:=A^{0}$. Then $-A$ generates a strongly continuous analytic semigroup on $L_{p}(M)$ and has the maximal regularity property on $L_{p}(M)$.

In order to facilitate the proof of Theorem 5.1 we precede it with a technical lemma. In this connection we identify $\rho^{\lambda}$ with the point-wise multiplication operator $u \mapsto \rho^{\lambda} u$.

Lemma 5.4. Let $(\mathcal{A}, \mathcal{B})$ be a $\rho$-regular uniformly $\rho$-elliptic boundary value problem on $M_{T}$ and $\lambda \in \mathbb{R}$. Then there exists another such pair $\left(\mathcal{A}^{\prime}, \mathcal{B}^{\prime}\right)$ such that

$$
(\mathcal{A}, \mathcal{B}) \circ \rho^{\lambda}=\rho^{\lambda} \circ\left(\mathcal{A}^{\prime}, \mathcal{B}^{\prime}\right) .
$$

Proof. (1) Note that

$$
(\mathcal{A}, \mathcal{B}) \circ \rho^{\lambda}=\rho^{\lambda} \circ(\mathcal{A}, \mathcal{B})+\left[(\mathcal{A}, \mathcal{B}), \rho^{\lambda}\right]
$$

where the commutator

$$
\left[(\mathcal{A}, \mathcal{B}), \rho^{\lambda}\right] u:=(\mathcal{A}, \mathcal{B})\left(\rho^{\lambda} u\right)-\rho^{\lambda}(\mathcal{A}, \mathcal{B}) u
$$

is given by $\left(\left[\mathcal{A}, \rho^{\lambda}\right],\left[\mathcal{B}, \rho^{\lambda}\right]\right)$ with

$$
\left[\mathcal{A}, \rho^{\lambda}\right] u=-2\left(a \cdot \operatorname{grad} \rho^{\lambda} \mid \operatorname{grad} u\right)+\left(\left(\vec{a} \mid \operatorname{grad} \rho^{\lambda}\right)-\operatorname{div}\left(a \cdot \operatorname{grad} \rho^{\lambda}\right)\right) u
$$

and

$$
\left[\mathcal{B}, \rho^{\lambda}\right] u=\left(0,\left(\nu \mid \gamma\left(a \cdot \operatorname{grad} \rho^{\lambda}\right)\right) u\right)
$$

For abbreviation, gl $:=\operatorname{grad} \log$. Then

$$
\operatorname{grad} \rho^{\lambda}=\lambda \rho^{\lambda-1} \operatorname{grad} \rho=\rho^{\lambda} \lambda \operatorname{gl} \rho .
$$


We set

$$
\begin{gathered}
\vec{a}^{\prime}:=-2 \lambda a \cdot \operatorname{gl} \rho, \quad a_{0}^{\prime}:=\lambda((\vec{a} \mid \operatorname{gl} \rho)-\operatorname{div}(a \cdot \operatorname{gl} \rho))-\lambda^{2}(a \cdot \operatorname{gl} \rho \mid \operatorname{gl} \rho), \\
b_{0}^{\prime}:=\lambda(\nu \mid \gamma(a \cdot \operatorname{gl} \rho)) .
\end{gathered}
$$

Moreover,

$$
\mathcal{A}^{\prime} u:=\mathcal{A} u+\left(\vec{a}^{\prime} \mid \operatorname{grad} u\right)+a_{0}^{\prime} u, \quad \mathcal{B}^{\prime} u:=\mathcal{B} u+\left(0, b_{0}^{\prime} \gamma u\right) .
$$

It follows from (5.6) and (5.7) that $\left(\mathcal{A}^{\prime}, \mathcal{B}^{\prime}\right)$ satisfies (5.5). Hence it remains to show that $\left(\vec{a}^{\prime}, a_{0}^{\prime}, b_{0}^{\prime}\right)$ possesses the same regularity properties as $\left(\vec{a}, a_{0}, b_{0}\right)$.

(2) We know from (4.3) and (4.4) that

$$
d \log \rho \in B C^{1}\left(T^{*} \hat{M}\right) \doteq B C^{1}\left(T^{*} M ; \rho\right) .
$$

Using this, $\operatorname{grad}=g^{\sharp} d$, and (A.8) it follows

$$
\operatorname{gl} \rho=g^{\sharp} d \log \rho \in B C^{1,2}(T M ; \rho) .
$$

It is now an easy consequence of this, the assumptions on $a$ and $\vec{a}$, and of A.5 that

$$
\vec{a}^{\prime} \in L_{\infty}(T M \times J), \quad(\vec{a} \mid \operatorname{gl} \rho) \in L_{\infty}\left(M_{T}\right) .
$$

From (5.8), A.9), and A.10 we infer

$$
\begin{aligned}
|\operatorname{div}(a \cdot \operatorname{gl} \rho)| & =|\nabla(a \cdot \operatorname{gl} \rho)|_{g_{1}^{1}} \\
& \leq \rho^{-1}|\nabla a|_{g_{1}^{2}} \rho|\operatorname{gl} \rho|_{g_{1}^{0}}+\rho^{-2}|a|_{g_{1}^{1}} \rho^{2}|\nabla \operatorname{gl} \rho|_{g_{1}^{1}}
\end{aligned}
$$

This guarantees that the second summand of $a_{0}^{\prime}$ belongs to $L_{\infty}\left(M_{T}\right)$. Similarly,

$$
|(a \cdot \operatorname{gl} \rho \mid \operatorname{gl} \rho)| \leq \rho^{-2}|a|_{g_{1}^{1}}\left(\rho|\operatorname{gl} \rho|_{g_{1}^{0}}\right)^{2}
$$

implies that the third summand lies in $L_{\infty}\left(M_{T}\right)$ as well. Hence, by the second part of (5.9),

$$
a_{0}^{\prime} \in L_{\infty}\left(M_{T}\right)
$$

Let $\hat{\nu}$ be the unit normal vector field of $\partial \hat{M}$. In local coordinates,

$$
\hat{\nu}=\left(\hat{g}_{11}\right)^{-1 / 2} \partial / \partial x^{1}=\rho\left(g_{11}\right)^{-1 / 2} \partial / \partial x^{1}=\rho \nu .
$$

Thus $\nu \in B C^{1,1}\left(T M_{\mid \partial M} ; \rho\right)$. This implies for the conormal field

$$
\nu_{b}=g_{b} \nu \in B C^{1,-1}\left(T^{*} M_{\mid \partial M} ; \rho\right) .
$$

As above, we derive from 5.8

$$
\gamma(a \cdot \operatorname{gl} \rho) \in B C^{(1,1 / 2)}\left(T M_{\mid \partial M} ; \rho\right) .
$$

Therefore, by (5.12),

$$
b_{0}^{\prime}=\lambda \nu_{b} \cdot \gamma(a \cdot \operatorname{gl} \rho) \in B C^{(1,1 / 2),-1}\left(\partial M_{T} ; \rho\right) .
$$

Now (5.9), (5.10), and (5.13) imply the assertion. 
Proof of Theorem 5.1thm.5.1, (1) By the definitions of $\hat{g}$ and the gradient we get

$$
\rho^{-2}\left(X \mid \operatorname{grad}_{\hat{g}} u\right)=\left(X \mid \operatorname{grad}_{\hat{g}} u\right)_{\hat{g}}=\langle d u, X\rangle=(X \mid \operatorname{grad} u)
$$

for any $C^{1}$ function $u$ and any vector field $X$ on $M$. From this we obtain

$$
\operatorname{grad} u=\rho^{-2} \operatorname{grad}_{\hat{g}} u \text {. }
$$

We also note that (A.2) A.4 imply

$$
|\cdot|_{\hat{g}_{\sigma}^{\tau}}=\rho^{\tau-\sigma}|\cdot|_{g_{\sigma}^{\tau}}, \quad \sigma, \tau \in \mathbb{N} .
$$

We put $\hat{a}:=\rho^{-2} a$. Then we infer from (5.16)

$$
|\hat{a}|_{\hat{g}_{1}^{1}}=\rho^{-2}|a|_{g_{1}^{1}} \text {. }
$$

Note that $\nabla \hat{a}=\rho^{-2} \nabla a-2(d \log \rho) \cdot \hat{a}($ cf. (5.7D) $)$. Hence, see (A.5),

$$
\begin{aligned}
\rho|\nabla \hat{a}|_{g_{1}^{2}} & \leq \rho^{-1}|\nabla a|_{g_{1}^{2}}+2 \rho|d \log \rho|_{g_{0}^{1}}|\hat{a}|_{g_{1}^{1}} \\
& =\rho^{-1}|\nabla a|_{g_{1}^{2}}+2|d \log \rho|_{\hat{g}_{0}^{1}}|\hat{a}|_{g_{1}^{1}},
\end{aligned}
$$

the last equality being a consequence of (5.16). From this, (4.3), (5.17), and the assumption on $a$ we deduce

$$
\hat{a} \in B C^{(1,1 / 2)}\left(T_{1}^{1} M \times J ; \rho\right) \doteq B C^{(1,1 / 2)}\left(T_{1}^{1} \hat{M} \times J\right) .
$$

By replacing the index $\mathrm{H}$ in (3.13) by $\hat{g}$ and using (5.14) and (5.15) we find

$$
\begin{aligned}
\operatorname{div}(a \cdot \operatorname{grad} u) & =\operatorname{div}\left(\rho^{2} \hat{a} \cdot \operatorname{grad} u\right) \\
& =\operatorname{div}_{\hat{g}}\left(\hat{a} \cdot \operatorname{grad}_{\hat{g}} u\right)+m(\rho \hat{a} \cdot \operatorname{grad} \rho \mid \operatorname{grad} u) \\
& =\operatorname{div}_{\hat{g}}\left(\hat{a} \cdot \operatorname{grad}_{\hat{g}} u\right)+\left(m \hat{a} \cdot \rho^{-1} \operatorname{grad}_{\hat{g}} \rho \mid \operatorname{grad}_{\hat{g}} u\right)_{\hat{g}} .
\end{aligned}
$$

Observe that

$$
\rho^{-1} \operatorname{grad}_{\hat{g}} \rho=\rho^{-1} \hat{g}^{\sharp} d \rho=\hat{g}^{\sharp} d \log \rho .
$$

Hence, by (A.5) and (A.8),

$$
\left|\hat{a} \cdot \rho^{-1} \operatorname{grad}_{\hat{g}} \rho\right|_{\hat{g}} \leq|\hat{a}|_{\hat{g}_{1}^{1}}|d \log \rho|_{\hat{g}_{0}^{1}} .
$$

This, (5.18), and (4.3) imply

$$
\hat{a} \cdot \rho^{-1} \operatorname{grad}_{\hat{g}} \rho \in L_{\infty}(T \hat{M} \times J) .
$$

Furthermore, by (5.15) and $\hat{g}=\rho^{-2} g$,

$$
(\vec{a} \mid \operatorname{grad} u)=\left(\vec{a} \mid \rho^{-2} \operatorname{grad}_{\hat{g}} u\right)=\left(\vec{a} \mid \operatorname{grad}_{\hat{g}} u\right)_{\hat{g}} .
$$

From (5.16) we derive

$$
\left\||\vec{a}|_{\hat{g}}\right\|_{\infty}=\left\|\rho^{-1}|\vec{a}|_{g}\right\|_{\infty}=\|\vec{a}\|_{L_{\infty}(T M \times J ; \rho)} .
$$

Thus it follows from t5.20 that

$$
\hat{d}:=m \hat{a} \cdot \rho^{-1} \operatorname{grad}_{\hat{g}} \rho+\vec{a} \in L_{\infty}(T \hat{M} \times J) .
$$

Now we put

$$
\hat{\mathcal{A}} u:=-\operatorname{div}_{\hat{g}}\left(\hat{a} \cdot \operatorname{grad}_{\hat{g}} u\right)+\left(\hat{d} \mid \operatorname{grad}_{\hat{g}} u\right)_{\hat{g}}+a_{0} u \text {. }
$$


Then (5.19) shows

$$
\mathcal{A} u=\hat{\mathcal{A}} u, \quad u \in W_{p}^{(2,1),-m / p}\left(M_{T} ; \rho\right) \doteq W_{p}^{(2,1)}\left(\hat{M}_{T}\right),
$$

the last equivalence being a consequence of (4.4).

(2) Recalling (5.11) and $\dot{\rho}=\rho \mid \partial M$, we find

$$
\begin{aligned}
(\nu \mid \gamma(a \cdot \operatorname{grad} u)) & =\left(\nu \mid \gamma\left(\hat{a} \cdot \operatorname{grad}_{\hat{g}} u\right)\right) \\
& =\dot{\rho}^{2}\left(\nu \mid \gamma\left(\hat{a} \cdot \operatorname{grad}_{\hat{g}} u\right)\right)_{\hat{g}}=\dot{\rho}\left(\hat{\nu} \mid \gamma\left(\hat{a} \cdot \operatorname{grad}_{\hat{g}} u\right)\right)_{\hat{g}} .
\end{aligned}
$$

We denote by $\dot{\nabla}$ the Levi-Civita connection of $\partial M$ and set $\hat{b}_{0}:=\dot{\rho}^{-1} b_{0}$. Then

$$
\dot{\nabla} \hat{b}_{0}=\dot{\rho}^{-1} \dot{\nabla} b_{0}-(d \log \dot{\rho}) \hat{b}_{0} .
$$

Relation (5.16) implies

$$
\dot{\rho}|d \log \dot{\rho}|_{\dot{g}_{0}^{1}}=|d \log \dot{\rho}|_{\hat{g}_{0}^{1}} .
$$

Since $b_{0} \in B C^{(1,1 / 2),-1}\left(\partial_{1} M_{T} ; \rho\right)$ it holds

$$
\begin{gathered}
\left\|\hat{b}_{0}\right\|_{L_{\infty}\left(\partial_{1} \hat{M}_{T}\right)}=\left\|\dot{\rho}^{-1} b_{0}\right\|_{L_{\infty}\left(\partial_{1} M_{T}\right)}<\infty, \\
\left\|\left|\dot{\nabla} b_{0}\right|_{\dot{g}_{0}^{1}}\right\|_{L_{\infty}\left(\partial_{1} M_{T}\right)}<\infty .
\end{gathered}
$$

Thus we get from (5.25), (5.26), Example 4.1(b), and (4.3) (applied to $\left.\partial_{1} M=\partial_{1} \hat{M}\right)$ that $\left\|\dot{\rho}\left|\dot{\nabla} \hat{b}_{0}\right|_{\dot{g}_{0}^{1}}\right\|_{L_{\infty}\left(\partial_{1} M_{T}\right)}$ is finite. This implies

$$
\hat{b}_{0} \in B C^{(1,1 / 2)}\left(\partial_{1} M_{T} ; \rho\right) \doteq B C^{(1,1 / 2)}\left(\partial_{1} \hat{M}_{T}\right) .
$$

Now we set

$$
\hat{\mathcal{B}}_{1} u:=\left(\hat{\nu} \mid \gamma\left(\hat{a} \cdot \operatorname{grad}_{\hat{g}} u\right)\right)_{\hat{g}}+\hat{b}_{0} \gamma u
$$

and $\hat{\mathcal{B}}:=\left(\mathcal{B}_{0}, \hat{\mathcal{B}}_{1}\right)$. Then we see from (5.18), (5.22), (5.27), and (5.1) that $(\hat{\mathcal{A}}, \hat{\mathcal{B}})$ is a regular uniformly elliptic boundary value problem on $\hat{M}_{T}$. Furthermore,

$$
\mathcal{B} u=\left(\hat{\mathcal{B}}_{0} u, \dot{\rho} \hat{\mathcal{B}}_{1} u\right) .
$$

(3) Suppose $\left(f, h, u_{0}\right) \in \mathcal{W}_{p, \mathrm{cc}}^{(2,1),-m / p}\left(M_{T} ; \rho\right)$. Then, by (4.7),

$$
\dot{\rho}^{-1} h_{1} \in W_{p}^{(1-1 / p)(1,1 / 2),-(m-1) / p}\left(\partial_{1} M_{T} ; \dot{\rho}\right) .
$$

More precisely, set $\hat{h}:=\left(h_{0}, \dot{\rho}^{-1} h_{1}\right)$. Then (4.7), (4.4), and (4.6) imply

$$
\left(\left(f, h, u_{0}\right) \mapsto\left(f, \hat{h}, u_{0}\right)\right) \in \mathcal{L} \operatorname{is}\left(\mathcal{W}_{p, \mathrm{cc}}^{(2,1),-m / p}\left(M_{T} ; \rho\right), \mathcal{W}_{p, \mathrm{cc}}^{(2,1)}\left(\hat{M}_{T}\right)\right) .
$$

In addition, we deduce from (5.23) and (5.28) that $u \in W_{p}^{(2,1)}\left(M_{T} ; \rho\right)$ is a solution of (3.1) iff $u \in W_{p}^{(2,1)}\left(\hat{M}_{T}\right)$ and $\left(\partial+\hat{\mathcal{A}}, \hat{\mathcal{B}}, \gamma_{0}\right) u=\left(f, \hat{h}, u_{0}\right)$. Now Theorem 3.1] implies the validity of the assertion if $\lambda=-m / p$.

(4) Let $\lambda \neq-m / p$. Lemma 5.4 guarantees the existence of a $\rho$-regular uniformly $\rho$-elliptic boundary value problem $\left(\mathcal{A}^{\prime}, \mathcal{B}^{\prime}\right)$ on $M_{T}$ such that

$$
\rho^{\lambda} \circ(\mathcal{A}, \mathcal{B})=\left(\mathcal{A}^{\prime}, \mathcal{B}^{\prime}\right) \circ \rho^{\lambda} \text {. }
$$

By (4.5) and (4.7) it follows that $\left(f, h, u_{0}\right) \in \mathcal{W}_{p, \mathrm{cc}}^{(2,1), \lambda}\left(M_{T} ; \rho\right)$ iff

$$
\left(f^{\prime}, h^{\prime}, u_{0}^{\prime}\right):=\rho^{\lambda+m / p}\left(f, h, u_{0}\right) \in \mathcal{W}_{p, \mathrm{cc}}^{(2,1),-m / p}\left(M_{T} ; \rho\right)
$$


and $u \in W_{p}^{(2,1), \lambda}\left(M_{T} ; \rho\right)$ iff

$$
u^{\prime}:=\rho^{\lambda+m / p} u \in W_{p}^{(2,1),-m / p}\left(M_{T} ; \rho\right) .
$$

From (5.29) we get

$$
\left(\mathcal{A}, \mathcal{B}, \gamma_{0}\right) u=\left(f, h, u_{0}\right) \Longleftrightarrow\left(\mathcal{A}^{\prime}, \mathcal{B}^{\prime}, \gamma_{0}\right) u^{\prime}=\left(f^{\prime}, h^{\prime}, u_{0}\right) .
$$

As the claim holds for $\lambda=-m / p$, the assertion follows.

Remarks 5.5. (a) It is obvious from this proof that there is a straightforward parameter-dependent analogue of the supplement to Theorem 3.1 for degenerate parabolic problems.

(b) Remarks 3.3 apply in the present setting also.

\section{Appendix: Tensor Bundles}

Let $M$ be a manifold and $V=(V, \pi, M)$ a vector bundle of rank $n$ over it. For a nonempty subset $S$ of $M$ we denote by $V_{\mid S}$ the restriction $\pi^{-1}(S)$ of $V$ to $S$. If $S$ is a submanifold or a union of connected components of $\partial M$, then $V_{\mid S}$ is a vector bundle of rank $n$ over $S$. As usual, $V_{p}:=V_{\{p\}}$ is the fibre $\pi^{-1}(p)$ of $V$ over $p$. By $\Gamma(S, V)$ we mean the $\mathbb{R}^{S}$ module of all sections of $V$ (no smoothness).

As usual, $T M$ and $T^{*} M$ are the tangent and cotangent bundles of $M$. Then $T_{\tau}^{\sigma} M:=T M^{\otimes \sigma} \otimes T^{*} M^{\otimes \tau}$ is for $\sigma, \tau \in \mathbb{N}$ the $(\sigma, \tau)$-tensor bundle of $M$, that is, the vector bundle of all tensors on $M$ being contravariant of order $\sigma$ and covariant of order $\tau$. In particular, $T_{0}^{1} M=T M$ and $T_{1}^{0} M=T^{*} M$, as well as $T_{0}^{0} M=M \times \mathbb{R}$.

For $\nu \in \mathbb{N}^{\times}$we put $\mathbb{J}_{\nu}:=\{1, \ldots, m\}^{\nu}$. Then, given local coordinates $\kappa=\left(x^{1}, \ldots, x^{m}\right)$ and setting

$$
\frac{\partial}{\partial x^{(i)}}:=\frac{\partial}{\partial x^{i_{1}}} \otimes \cdots \otimes \frac{\partial}{\partial x^{i_{\sigma}}}, \quad d x^{(j)}:=d x^{j_{1}} \otimes \cdots \otimes d x^{j_{\tau}}
$$

for $(i)=\left(i_{1}, \ldots, i_{\sigma}\right) \in \mathbb{J}_{\sigma},(j) \in \mathbb{J}_{\tau}$, the local representation of a $(\sigma, \tau)$-tensor field $a \in \Gamma\left(T_{\tau}^{\sigma} M\right)$ with respect to these coordinates is given by

$$
a=a_{(j)}^{(i)} \frac{\partial}{\partial x^{(i)}} \otimes d x^{(j)}
$$

with $a_{(j)}^{(i)} \in \mathbb{R}^{U_{\kappa}}$. We use the summation convention for (multi-)indices labeling coordinates or bases. Thus such a repeated index, which appears once as a superscript and once as a subscript, implies summation over its whole range.

Suppose $\sigma_{1}, \sigma_{2}, \tau_{1}, \tau_{2} \in \mathbb{N}$. Then the complete contraction

$$
\Gamma\left(T_{\tau_{2}+\sigma_{1}}^{\sigma_{2}+\tau_{1}} M\right) \times \Gamma\left(T_{\tau_{1}}^{\sigma_{1}} M\right) \rightarrow \Gamma\left(T_{\tau_{2}}^{\sigma_{2}} M\right), \quad(a, b) \mapsto a \cdot b
$$

is defined as follows: Given $\left(i_{k}\right) \in \mathbb{J}_{\sigma_{k}}$ and $\left(j_{k}\right) \in \mathbb{J}_{\tau_{k}}$ for $k=1$, 2, we set

$$
\left(i_{2} ; j_{1}\right):=\left(i_{2,1}, \ldots, i_{2, \sigma_{2}}, j_{1,1}, \ldots, j_{1, \tau_{1}}\right) \in \mathbb{J}_{\sigma_{2}+\tau_{1}}
$$


etc., using obvious interpretations if $\min \{\sigma, \tau\}=0$. Suppose $a \in \Gamma\left(T_{\tau_{2}+\sigma_{1}}^{\sigma_{2}+\tau_{1}} M\right)$ and $b \in \Gamma\left(T_{\tau_{1}}^{\sigma_{1}} M\right)$ are locally represented on $U_{\kappa}$ by

$$
a=a_{\left(j_{2} ; i_{1}\right)}^{\left(i_{2} ; j_{1}\right)} \frac{\partial}{\partial x^{\left(i_{2}\right)}} \otimes \frac{\partial}{\partial x^{\left(j_{1}\right)}} \otimes d x^{\left(j_{2}\right)} \otimes d x^{\left(i_{1}\right)}, \quad b=b_{\left(j_{1}\right)}^{\left(i_{1}\right)} \frac{\partial}{\partial x^{\left(i_{1}\right)}} \otimes d x^{\left(j_{1}\right)} .
$$

Then the local representation of $a \cdot b$ on $U_{\kappa}$ is given by

$$
a_{\left(j_{2} ; i_{1}\right)}^{\left(i_{2} ; j_{1}\right)} b_{\left(j_{1}\right)}^{\left(i_{1}\right)} \frac{\partial}{\partial x^{\left(i_{2}\right)}} \otimes d x^{\left(j_{2}\right)}
$$

Let $g$ be a Riemannian metric on $T M$. We write $g_{b}: T M \rightarrow T^{*} M$ for the (fiber-wise defined) Riesz isomorphism. Thus $\left\langle g_{b} X, Y\right\rangle=g(X, Y)$ for $X, Y \in \Gamma(T M)$, where $\langle\cdot, \cdot\rangle: \Gamma\left(T^{*} M\right) \times \Gamma(T M) \rightarrow \mathbb{R}^{M}$ is the natural (fiberwise defined) duality pairing. The inverse of $g_{b}$ is denoted by $g^{\sharp}$. Then $g^{*}$, the adjoint Riemannian metric on $T^{*} M$, is defined by $g^{*}(\alpha, \beta):=g\left(g^{\sharp} \alpha, g^{\sharp} \beta\right)$ for $\alpha, \beta \in \Gamma\left(T^{*} M\right)$. In local coordinates

$$
g=g_{i j} d x^{i} \otimes d x^{j}, \quad g^{*}=g^{i j} \frac{\partial}{\partial x^{i}} \otimes \frac{\partial}{\partial x^{j}},
$$

$\left[g^{i j}\right]$ being the inverse of the $(m \times m)$-matrix $\left[g_{i j}\right]$.

The metric $g$ induces a vector bundle metric on $T_{\tau}^{\sigma} M$ which we denote by $g_{\sigma}^{\tau}$. In local coordinates

$$
g_{\sigma}^{\tau}(a, b)=g_{(i)(j)} g^{(k)(\ell)} a_{(k)}^{(i)} b_{(\ell)}^{(j)}, \quad a, b \in \Gamma\left(T_{\tau}^{\sigma} M\right),
$$

where

$$
g_{(i)(j)}:=g_{i_{1} j_{1}} \cdots g_{i_{\sigma} j_{\sigma}}, \quad g^{(k)(\ell)}:=g^{k_{1} \ell_{1}} \cdots g^{k_{\tau} \ell_{\tau}}
$$

for $(i),(j) \in \mathbb{J}_{\sigma}$ and $(k),(\ell) \in \mathbb{J}_{\tau}$. Note $g_{1}^{0}=g$ and $g_{0}^{1}=g^{*}$ and $g_{0}^{0}(a, b)=a b$ for $a, b \in \Gamma(M \times \mathbb{R})=\mathbb{R}^{M}$. Moreover,

$$
|\cdot|_{g_{\sigma}^{\tau}}: \Gamma\left(T_{\tau}^{\sigma} M\right) \rightarrow\left(\mathbb{R}^{+}\right)^{M}, \quad a \mapsto \sqrt{g_{\sigma}^{\tau}(a, a)}
$$

is the vector bundle norm on $T_{\tau}^{\sigma} M$ induced by $g$. It follows that the complete contraction satisfies

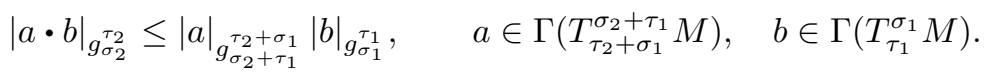

We define a vector bundle isomorphism $T_{\tau+1}^{\sigma} M \rightarrow T_{\tau}^{\sigma+1} M, a \mapsto a^{\sharp}$ by $a^{\sharp}\left(\alpha_{1}, \ldots, \alpha_{\sigma}, \alpha, X_{1}, \ldots, X_{\tau}\right):=a\left(\alpha_{1}, \ldots, \alpha_{\sigma}, X_{1}, \ldots, X_{\tau}, g^{\sharp} \alpha\right)$

for $X_{1}, \ldots, X_{\tau} \in \Gamma(T M)$ and $\alpha, \alpha_{1}, \ldots, \alpha_{\sigma} \in \Gamma\left(T^{*} M\right)$. If $a_{(j ; k)}^{(i)}$ with $(i) \in \mathbb{J}_{\sigma}$, $(j) \in \mathbb{J}_{\tau}$, and $k \in \mathbb{J}_{1}$ is the coefficient of $a$ in a local coordinate representation, then

This implies

$$
\left(a^{\sharp}\right)_{(j)}^{(i ; k)}=g^{k \ell} a_{(j ; \ell)}^{(i)} .
$$

$$
\left|a^{\sharp}\right|_{\sigma+1}^{\tau}=|a|_{g_{\sigma}^{\tau+1}} .
$$

The Levi-Civita connection on $T M$ is denoted by $\nabla=\nabla_{g}$. We use the same symbol for its natural extension to a metric connection on $T_{\tau}^{\sigma} M$. Then the corresponding covariant derivative is the linear map

$$
\nabla: C^{\infty}\left(T_{\tau}^{\sigma} M\right) \rightarrow C^{\infty}\left(T_{\tau+1}^{\sigma} M\right), \quad a \mapsto \nabla a,
$$


defined by $\langle\nabla a, b \otimes X\rangle:=\left\langle\nabla_{X} a, b\right\rangle$ for $b \in C^{\infty}\left(T_{\sigma}^{\tau} M\right)$ and $X \in C^{\infty}(T M)$. It is a well-defined continuous linear map from $C^{1}\left(T_{\tau}^{\sigma} M\right)$ into $C\left(T_{\tau+1}^{\sigma} M\right)$, as follows from its local representation. For $k \in \mathbb{N}$ we define

$$
\nabla^{k}: C^{k}\left(T_{\tau}^{\sigma} M\right) \rightarrow C\left(T_{\tau+k}^{\sigma} M\right), \quad a \mapsto \nabla^{k} a
$$

by $\nabla^{0} a:=a$ and $\nabla^{k+1}:=\nabla \circ \nabla^{k}$.

In local coordinates $\kappa=\left(x^{1}, \ldots, x^{m}\right)$ the volume measure $d v=d v_{g}$ of $(M, g)$ is represented by $\kappa_{*} d v=\kappa_{*} \sqrt{g} d x$, where $\sqrt{g}:=\left(\operatorname{det}\left[g_{i j}\right]\right)^{1 / 2}$ and $d x$ is the Lebesgue measure on $\mathbb{R}^{m}$.

The contraction $\mathrm{C}: T_{\tau+1}^{\sigma+1} M \rightarrow T_{\tau}^{\sigma} M, a \mapsto \mathrm{C} a$ is given in local coordinates by $(\mathrm{C} a)_{(j)}^{(i)}:=a_{(j ; k)}^{(i ; k)}$. It follows

$$
|\mathrm{C} a|_{g_{\sigma}^{\tau}}=|a|_{g_{\sigma+1}^{\tau+1}}
$$

Recall that the divergence of tensor fields is the map

$$
\operatorname{div}=\operatorname{div}_{g}: C^{1}\left(T_{\tau}^{\sigma+1} M\right) \rightarrow C\left(T_{\tau}^{\sigma} M\right), \quad a \mapsto \operatorname{div} a:=\mathrm{C}(\nabla a) .
$$

If $X$ is a $C^{1}$ vector field on $M$, then $\operatorname{div} X$ has the well-known local representation

$$
\frac{1}{\sqrt{g}} \frac{\partial}{\partial x^{i}}\left(\sqrt{g} X^{i}\right), \quad X=X^{i} \frac{\partial}{\partial x^{i}} .
$$

The gradient, $\operatorname{grad} u=\operatorname{grad}_{g} u$, of a $C^{1}$ function $u$ is the continuous vector field $g^{\sharp} d u$.

Suppose $a \in C^{1}\left(T_{1}^{1} M\right)$. Then, in terms of covariant derivatives,

$$
\operatorname{div}(a \operatorname{grad} u)=a^{\sharp} \cdot \nabla^{2} u+\operatorname{div}\left(a^{\sharp}\right) \cdot \nabla u \text {. }
$$

\section{References}

[1] H. Amann. Linear and Quasilinear Parabolic Problems, Volume I: Abstract Linear Theory. Birkhäuser, Basel, 1995.

[2] H. Amann. Maximal regularity for nonautonomous evolution equations. Adv. Nonl. Studies, 4 (2004), 417-430.

[3] H. Amann. Quasilinear parabolic problems via maximal regularity. Advances in Diff. Equ., 10(10) (2005), 1081-1110.

[4] H. Amann. Anisotropic Function Spaces and Maximal Regularity for Parabolic Problems. Part 1: Function Spaces. Jindřich Nečas Center for Mathematical Modeling, Lecture Notes, 6, Prague, 2009, Corrections and Remarks to 'Anisotropic Function Spaces and Maximal Regularity for Parabolic Problems. Part 1: Function Spaces', www.math.uzh.ch/amann.

[5] H. Amann. Anisotropic function spaces on singular manifolds, 2012. arXiv: 1204.0606.

[6] H. Amann. Function spaces on singular manifolds. Math. Nachr., 286 (2012), $436-475$.

[7] H. Amann. Parabolic equations on noncompact Riemannian manifolds. In preparation.

[8] H. Amann. Pseudodifferential boundary value problems. In preparation. 
[9] H. Amann. Uniformly regular and singular Riemannian manifolds. In preparation.

[10] B. Ammann, A.D. Ionescu, V. Nistor. Sobolev spaces on Lie manifolds and regularity for polyhedral domains. Doc. Math., 11 (2006), 161-206.

[11] B. Ammann, R. Lauter, V. Nistor. On the geometry of Riemannian manifolds with a Lie structure at infinity. Int. J. Math. Math. Sci., (2004), 161-193.

[12] B. Ammann, V. Nistor. Weighted Sobolev spaces and regularity for polyhedral domains. Comput. Methods Appl. Mech. Engrg., 196 (2007), 3650-3659.

[13] C. Bacuta, A.L. Mazzucato, V. Nistor. Anisotropic regularity and optimal rates of convergence for the finite element method on three dimensional polyhedral domains, 2012. arXiv:1205.2128

[14] C. Bandle, F. Punzo, A. Tesei. Existence and nonexistence of patterns on Riemannian manifolds. J. Math. Anal. Appl., 387(1) (2012), 33-47.

[15] V. Barbu, A. Favini, S. Romanelli. Degenerate evolution equations and regularity of their associated semigroups. Funkcial. Ekvac., 39(3) (1996), 421-448.

[16] E.B. Davies. Heat Kernels and Spectral Theory. Cambridge Univ. Press, Cambridge, 1989.

[17] R. Denk, M. Hieber, J. Prüss. $\mathcal{R}$-boundedness, Fourier multipliers and problems of elliptic and parabolic type. Mem. Amer. Math. Soc., 166(788) (2003).

[18] G. Dore. $L^{p}$ regularity for abstract differential equations. In Functional analysis and related topics, 1991 (Kyoto), Lecture Notes in Math., 1540, pages 25-38. Springer-Verlag, Berlin, 1993.

[19] S. Fornaro, G. Metafune, D. Pallara. Analytic semigroups generated in $L^{p}$ by elliptic operators with high order degeneracy at the boundary. Note Mat., 31(1) (2011), 103-116.

[20] G. Fragnelli, G. Ruiz Goldstein, J.A. Goldstein, S. Romanelli. Generators with interior degeneracy on spaces of $L^{2}$ type. Electron. J. Differential Equations, (2012), No. 189, 1-30.

[21] A. Grigor'yan. Heat Kernel and Analysis on Manifolds. American Mathematical Society, Providence, RI, 2009.

[22] G. Grubb. Parameter-elliptic and parabolic pseudodifferential boundary problems in global $L_{p}$ Sobolev spaces. Math. Z., 218 (1995), 43-90.

[23] G. Grubb. Functional Calculus of Pseudodifferential Boundary Problems. Birkhäuser, Boston, MA, 1996.

[24] G. Grubb, N.J. Kokholm. A global calculus of parameter-dependent pseudodifferential boundary problems in $L_{p}$ Sobolev spaces. Acta Math., 171 (1993), $165-229$.

[25] V.A. Kondrat'ev. Boundary value problems for elliptic equations in domains with conical or angular points. Trudy Moskov. Mat. Ob̌̌č, 16 (1967), 209-292.

[26] P.Ch. Kunstmann, L. Weis. Maximal $L_{p}$-regularity for parabolic equations, Fourier multiplier theorems and $H^{\infty}$-functional calculus. In Functional analytic methods for evolution equations, Lecture Notes in Math., 1855, pages 65-311. Springer-Verlag, Berlin, 2004.

[27] O.A. Ladyzhenskaya, V.A. Solonnikov, N.N. Ural'ceva. Linear and Quasilinear Equations of Parabolic Type. Amer. Math. Soc., Transl. Math. Monographs, Providence, R.I., 1968. 
[28] A. Lunardi. Analytic Semigroups and Optimal Regularity in Parabolic Problems. Birkhäuser, Basel, 1995.

[29] A.L. Mazzucato, V. Nistor. Mapping properties of heat kernels, maximal regularity, and semi-linear parabolic equations on noncompact manifolds. J. Hyperbolic Differ. Equ., 3(4) (2006), 599-629.

[30] M.A. Pozio, F. Punzo, A. Tesei. Criteria for well-posedness of degenerate elliptic and parabolic problems. J. Math. Pures Appl. (9), 90(4) (2008), 353-386.

[31] F. Punzo. On well-posedness of semilinear parabolic and elliptic problems in the hyperbolic space. J. Differential Equations, 251(7) (2011), 1972-1989.

[32] F. Punzo. Blow-up of solutions to semilinear parabolic equations on Riemannian manifolds with negative sectional curvature. J. Math. Anal. Appl., 387(2) (2012), 815-827.

[33] Y. Shao, G. Simonett. Continuous maximal regularity on uniformly regular Riemannian manifolds, 2013. In preparation.

[34] V. Vespri. Analytic semigroups, degenerate elliptic operators and applications to nonlinear Cauchy problems. Ann. Mat. Pura Appl. (4), 155 (1989), 353-388.

[35] Qi S. Zhang. Nonlinear parabolic problems on manifolds, and a nonexistence result for the noncompact Yamabe problem. Electron. Res. Announc. Amer. Math. Soc., 3 (1997), 45-51.

[36] Qi S. Zhang. Semilinear parabolic problems on manifolds and applications to the non-compact Yamabe problem. Electron. J. Differential Equations, (2000), No. $46,1-30$.

Herbert Amann

Math. Institut

Universität Zürich

Winterthurerstr. 190

CH 8057 Zürich

Switzerland

e-mail: herbert.amann@math.uzh.ch 